\title{
Transcranial direct current stimulation (tDCS) in the treatment of depression: Systematic review and meta-analysis of efficacy and tolerability
}

Article

Accepted Version

Creative Commons: Attribution-Noncommercial-No Derivative Works 4.0

Meron, D., Hedger, N., Garner, M. and Baldwin, D. S. (2015) Transcranial direct current stimulation (tDCS) in the treatment of depression: Systematic review and meta-analysis of efficacy and tolerability. Neuroscience and Biobehavioral Reviews, 57. pp. 46-62. ISSN 0149-7634 doi:

https://doi.org/10.1016/j.neubiorev.2015.07.012 Available at https://centaur.reading.ac.uk/87284/

It is advisable to refer to the publisher's version if you intend to cite from the work. See Guidance on citing.

To link to this article DOI: http://dx.doi.org/10.1016/j.neubiorev.2015.07.012

Publisher: Elsevier

All outputs in CentAUR are protected by Intellectual Property Rights law, including copyright law. Copyright and IPR is retained by the creators or other copyright holders. Terms and conditions for use of this material are defined in the End User Agreement. 


\section{www.reading.ac.uk/centaur}

\section{CentAUR}

Central Archive at the University of Reading

Reading's research outputs online 


\section{Accepted Manuscript}

Title: Transcranial direct current stimulation (tDCS) in the treatment of depression: systematic review and meta-analysis of efficacy and tolerability

Author: Daniel Meron Nicholas Hedger Matthew Garner David S. Baldwin

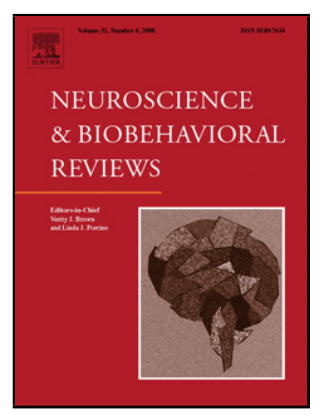

PII: S0149-7634(15)00197-9

DOI:

Reference: http://dx.doi.org/doi:10.1016/j.neubiorev.2015.07.012 NBR 2237

To appear in:

Received date: $\quad$ 12-2-2015

Revised date: 24-7-2015

Accepted date: $\quad$ 26-7-2015

Please cite this article as: Meron, D., Hedger, N., Garner, M., Baldwin, D.S., Transcranial direct current stimulation (tDCS) in the treatment of depression: systematic review and meta-analysis of efficacy and tolerability, Neuroscience and Biobehavioral Reviews (2015), http://dx.doi.org/10.1016/j.neubiorev.2015.07.012

This is a PDF file of an unedited manuscript that has been accepted for publication. As a service to our customers we are providing this early version of the manuscript. The manuscript will undergo copyediting, typesetting, and review of the resulting proof before it is published in its final form. Please note that during the production process errors may be discovered which could affect the content, and all legal disclaimers that apply to the journal pertain. 


\section{Highlights}

- We identified 10 RCTs $(N=393)$ of tDCS as treatment for MDE

- tDCS was superior to sham tDCS $(\mathrm{k}=11, \underline{g}=0.30,95 \% \mathrm{Cl}=[0.04,0.57], \underline{p}=.027)$

- $N=346$ to detect ( $80 \%$ power) this effect $(N=49 / N=12693$ for bounds of $\mathrm{Cl})$

- Current data do not support the use of tDCS in treatment resistant depression

- Larger studies over longer periods of treatment are needed 
Transcranial direct current stimulation (tDCS) in the treatment of depression: systematic review and metaanalysis of efficacy and tolerability

Daniel Meron ${ }_{a, b}$, Nicholas Hedger, Matthew Garner ${ }_{a, c}$, David S. Baldwin ${ }_{a, d}$

a. Clinical and Experimental Sciences, Faculty of Medicine, University of Southampton, University Department of Psychiatry, Academic Centre, College Keep, 4-12 Terminus Terrace, Southampton SO14 3DT United Kingdom. Tel. +442380718 520. Fax +442380718532

b. Avon \& Wiltshire Partnership NHS Trust, Jenner House, Langley Park, Chippenham, Wiltshire, SN15 1GG UK. c. Psychology, Faculty of Social \& Human Sciences, University of Southampton

d. Mood and Disorders Service, Southern Health NHS Foundation Trust, Southampton, UK

\author{
Author email addresses: \\ D. Meron: dan@soton.ac.uk \\ NAA Hedger : nick.hedger@soton.ac.uk \\ M Garner: M.J.GARNER@soton.ac.uk \\ DS Baldwin: D.S.Baldwin@soton.ac.uk
}

Corresponding author: Professor David Baldwin

\begin{abstract}
Background : Transcranial direct current stimulation (tDCS) is a potential alternative treatment option for major depressive episodes (MDE). Objectives: We address the efficacy and safety of tDCS in MDE. Methods: The outcome measures were Hedges' $g$ for continuous depression ratings, and categorical response and remission rates. Results: A random effects model indicated that tDCS was superior to sham tDCS $(k=11, N=393, g=0.30$, $95 \% \mathrm{Cl}=[0.04,0.57], p=.027)$. Adjunctive antidepressant medication and cognitive control training, negatively impacted on the treatment effect. The pooled log odds ratios (LOR) for response and remission were positive, but statistically non-significant (response: $k=9, L O R=0.36,95 \% \mathrm{CI}[-0.16,0.88], p=.176$, remission: $k=9, L O R=$ $0.25,95 \% \mathrm{Cl}[-0.42,0.91], p=.468)$. We estimated that for a study to detect the pooled continuous effect $(g=0.30)$ at $80 \%$ power (alpha $=.05)$, a total $N$ of at least 346 would be required (with the total $N$ required to detect the upper and lower bound being 49 and 12693 respectively).
\end{abstract}


Conclusions: tDCS may be efficacious for treatment of MDE. The data do not support the use of tDCS in treatment-resistant depression, or as an add-on augmentation treatment . Larger studies over longer treatment periods are needed.

Key words: Depression, meta-analysis, non-pharmacological therapies, systematic review, transcranial direct current stimulation, tDCS, Response, Remission, Cognitive Control Training.

\section{BACKGROUND}

Depressive disorders are prevalent, recurrent, often run a chronic course, and are associated with significant worldwide morbidity and mortality ${ }^{2,3}$. Treatment with antidepressant medication is often suboptimal in terms of efficacy, safety and tolerability ${ }^{4,5}$. Psychological interventions are associated with significant rates of suboptimal effectiveness, even when combined with antidepressant medication ${ }^{6}$. Electro-Convulsive Therapy (ECT) is highly effective, but is associated with significant stigma, and adverse effects ${ }^{6}$. Other invasive and non-invasive neurostimulation modalities have been proposed for the treatment of depression, but their utility may be limited by issues such as cost, tolerability and availability ${ }^{7}$. In particular, there are important differences between tDCS and repetitive transcranial magnetic stimulation (rTMS) in terms of adverse effect profiles, focality of stimulation, and also in the cost, availability and portability of equipment ${ }^{7}$. Trans-cranial direct current stimulation (tDCS) is a novel treatment modality for depression, which may represent an alternative to pharmacological or psychological treatments. tDCS is a non-invasive brain stimulation modality, which changes cortical tissue 'excitability' as a result of applying a weak $(0.5-2 \mathrm{~mA})$ direct current via scalp electrodes overlying targeted cortical areas. In contrast to other neurostimulation modalities, tDCS does not directly trigger action potentials in neuronal cells, but instead changes overall tissue excitability, and therefore may be more aptly regarded as a 'neuro-modulatory' rather than a neuro-stimulatory approach ${ }^{7}$. Cortical tissue underlying the anode (positive electrode) becomes hypo-polarized, and therefore hyper-excitable; areas underlying the cathode (negative electrode) become less excitable as the average resting potential becomes more polarized. The magnitude of these membrane polarization changes is not in itself sufficient to directly cause neurons to fire ${ }^{8}$. These effects continue after electrical stimulation ceases, and a single application can be associated with tissue excitability changes lasting more than 60 minutes ${ }^{9,10}$. These findings suggest tDCS is likely to be associated not only with transient membrane polarization changes, but also with longer-lasting synaptic changes ${ }^{11}$. 
The body of research describing the efficacy, safety and tolerability of tDCS in depression is growing. Three earlier meta-analyses have been published ${ }^{12-14}$, these used different methodologies and produced inconsistent findings. We therefore performed a systematic review and meta-analysis of the efficacy and tolerability of tDCS in depression, using a comprehensive set of meta-analytic tools, and incorporating all published randomised controlled trials to date.

\section{METHOD}

A literature search and meta analysis were conducted following the recommendations of the Cochrane collaboration $^{15}$ and the PRISMA guidelines ${ }^{1}$. Two authors (DM and $\mathrm{NH}$ ) performed the systematic review and data extraction. All discrepancies were resolved by consensus.

\subsection{LITERATURE REVIEW}

We searched the PubMed database using the following search strategy:

(((((“direct”[Title/Abstract]) AND “stimulation”[Title/Abstract])) OR “tdcs”[Title/Abstract])) AND

((“rand*”[Title/Abstract]) OR “control*”)) AND “depress*"[Title/Abstract]. The date range extended up to April 30"t 2015. We also scrutinized the reference lists in published meta-analyses of tDCS in depression and articles listed as citing these meta-analyses ${ }^{12-14}$.

Inclusion criteria used were: English language publications; Randomised, sham-controlled trials. Including data enabling calculation of effect size for depression rating scale change, and/or response/remission rates. Patient population with depressive disorders. tDCS as monotherapy or augmentation therapy for treatment of depression. Exclusion criteria used were: Studies in animals. Non-controlled or non-randomised trials. Case reports / case series. Trials of treatments for disorders other than depression. Trials of interventions other than tDCS. Duplicated data-sets.

\subsection{DATA EXTRACTION}

The following data were extracted: Population demographics including sample size. Diagnosis (unipolar/bipolar depression). tDCS characteristics (including number of sessions, montage, current used, inter-session intervals, sham stimulation characteristics). Efficacy outcome measures and outcomes (Including rating scale score changes and response/remission rates). Acceptability (using dropout numbers as a proxy measure). 
Outcome measures included both continuous depression rating scale scores, and categorical response/remission rates - we included both types of outcome for the following reasons: First, previous meta-analyses utilised continuous outcome measures ${ }^{12}$, categorical outcome measures ${ }^{13}$, or both ${ }^{14}$, which may explain the inconclusive and at times contradictory nature of their findings. Second, whereas continuous outcome measures may offer superior sensitivity, their specificity is considered inferior to that of categorical outcome measures. Third, while categorical response/remission rates may be more clinically 'meaningful', they require larger sample sizes, and as all studies included in our meta-analysis utilised continuous primary outcome measures, they may have lacked sufficient statistical power to reliably test hypotheses based on categorical outcomes.

We therefore extracted the following data: for continuous outcomes we meta-analysed depression rating scale scores at randomised blinded treatment endpoint, using the study primary outcome measure rating scale; for categorical outcomes, we meta-analysed remission and response rates for active and sham groups at randomised blinded treatment endpoint. Response was defined as $\geq 50 \%$ reduction in depression rating scale score from baseline to endpoint; we used the specified remission criteria provided by each study.

\subsection{META ANALYSIS}

Our adopted meta-analytic approach makes several important contributions to the literature (Table 2). We:

1. increase the number of included RCTs and subjects, compared to previous meta-analyses.

2. use a methodology combining continuous outcome measures (rating scale scores) with possibly more clinically relevant dichotomous measures (i.e. response and remission rates).

3. perform moderator analyses to clarify the effect of putative moderators identified in narrative analysis.

4. perform power and precision analyses to inform future research in terms of sample size planning.

5. identify important gaps in knowledge and suggest new directions for future research, methodological improvements and improved reporting standards.

6. Examine the use of tDCS in conjunction with antidepressant medication and with Cognitive Control Training (CCT)

7. clarify in which conditions tDCS might be clinically useful.

The primary effect size index used to quantify the continuous treatment effect was Hedge's $g$ - the difference in the reduction in depression severity rating scale scores (MADRS and/or HDRS) between the two groups (active 
We performed two additional meta-analyses to quantify the overall treatment effect in terms of categorical response and remission rates ${ }^{4}$. In both cases, we compared the differences between treatment and control groups by using the log odds ratio as an effect size index. A positive score represents a higher likelihood of response or remission in the treatment (active tDCS) group, relative to the control (sham tDCS) group. Response was defined as $50 \%$ reduction in depression rating scale score from baseline to endpoint. We used the remission criteria provided by each study.

As previous meta-analyses have indicated considerable heterogeneity in effect size estimates between studies ${ }^{12}$, we made an a priori decision to analyse our effect size data using a random effects model, due to its tolerance of heterogeneous effect sizes and conservative nature of estimation ${ }^{16,17}$. The random effects model assumes that each study estimates different values from a distribution of population parameters, rather than assuming studies are direct replications of each other. We assessed heterogeneity across effect sizes by using Cochran's $Q$ and $I^{2}$ statistics. Unless reported otherwise, parameter estimates were obtained via restricted maximum likelihood estimation, due to its accuracy relative to other estimators with smaller numbers of studies ${ }^{18}$. Statistical tests of model coefficients were computed via Wald-type chi squared tests. We additionally used a pseudo- $R^{2}$ statistic to assess the amount of heterogeneity between effects explained by including moderators ${ }^{19} \cdot R^{2}$ estimates the proportional reduction in heterogeneity after including moderators. For interpretation, it is important to note that this pseudo- $R^{2}$ does not include sampling variability, meaning that it is possible to get very large $R^{2}$ values, even 
when there are discrepancies between the model and the observed effects (provided these are not larger than expected by sampling variability). Model comparisons were conducted via likelihood ratio tests. All meta analyses were performed using the "metafor" package in $\mathrm{R}^{20}$.

To account for heterogeneity across treatment effects, we assessed the impact of potential categorical and continuous moderators of the treatment effect. The moderators we examined are listed in table 1 . Moderator data for each study were recorded in a structured fashion. Where the information was reported, we recorded both categorical moderators and continuous moderators. Two experienced authors ( $\mathrm{NH}, \mathrm{DM})$ acted together as coders, and no disagreements on coding decisions were encountered. 


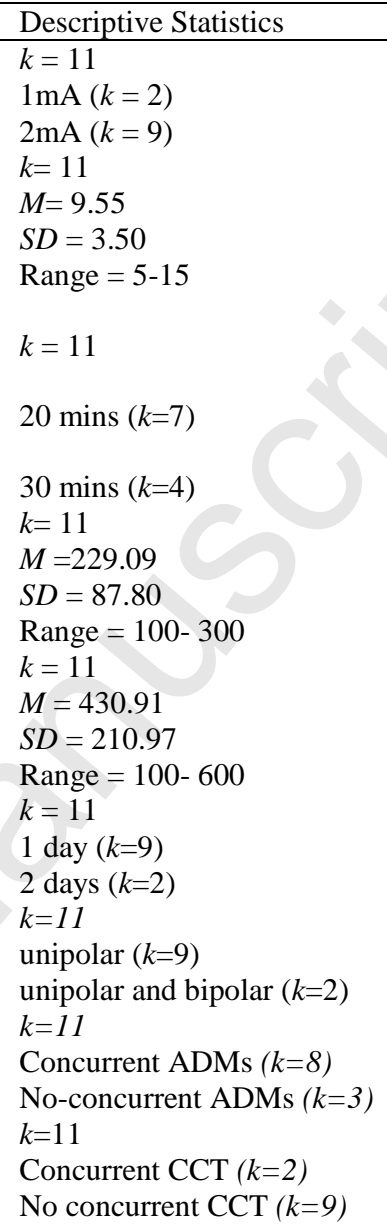

$k=11$

$M=229.09$

$S D=87.80$

Range $=100-300$

$k=11$

$M=430.91$

$S D=210.97$

Range $=100-600$

$k=11$

1 day $(k=9)$

2 days $(k=2)$

$k=11$

unipolar $(k=9)$

unipolar and bipolar $(k=2)$

$k=11$

Concurrent ADMs $(k=8)$

No-concurrent ADMs $(k=3)$

$k=11$

Concurrent CCT $(k=2)$

No concurrent CCT $(k=9)$

Number of sessions $x$ session duration (minutes)

$k=9$ (see table 3$)$

Table 1: variables examined as potential moderators of tDCS outcomes in depression. These include both participant-related and tDCS-related factors, with a view to enable meta-analytical examination of the variables found to be associated with tDCS outcomes in the narrative analysis. 


\section{Additional analyses: publication bias, precision and power}

To assess the impact of the so-called "file drawer problem", whereby unpublished null results can distort metaanalytic estimates, we performed 'fail-safe $N$ ' analyses using the methods developed by Orwin ${ }^{21}$ and Rosenthal ${ }^{22}$. This allowed estimation of the total number of unpublished studies averaging null effects that would be required to reduce the reported treatment effect size to target levels ${ }^{21}$ or to statistical non-significance ${ }^{22}$. Despite the existence of other meta-analyses estimating the magnitude of tDCS efficacy, to date there has been no formal, explicit attempt to use these parameter estimates to inform future research planning. We hence addressed this issue from both an Accuracy In Parameter Estimation (AIPE), and power analytic perspective. The AIPE analyses were used to estimate the appropriate sample size for future trials such that the expected width of the confidence interval meets a desired level of precision ${ }^{16}$. Unlike power analysis, this approach negates the need to invoke a null hypothesis significance test, and instead is solely concerned with precision in parameter estimation. This was achieved by using the non-central $t$ method described by Kelley ${ }^{23}$, implemented in the "MBESS" package in $\mathrm{R}^{24}$. To provide an assessment of the statistical power of individual studies, we estimated the power of each study to detect the summary effect estimated in the meta-analysis at a nominal level of significance $(p=.05)$. By extension, for future sample size planning, we calculated the minimum $N$ required to achieve adequate (80\%) power to detect the estimated summary effect.

\section{RESULTS: EFFICACY}

3.1 Randomized Controlled Trials (RCTs): We identified ten randomized controlled trials of tDCS in depression using PRISMA methodology (Figure 1a), and quality-assessed the each trial using the Cochrane Collaboration's tool for assessing risk of bias in randomised trials ${ }^{25}$ - this yielded an acceptable profile of Risk of Bias across the RCTs included in the meta-analysis (Figure 1b). Due to the diversity of study designs, we made the following decisions about the analysis of individual studies: 1 . Boggio et al. (2008) ${ }^{26}$ randomised participants to 3 groups: active tDCS over left DLPFC, sham tDCS over left DLPFC, and active occipital tDCS; we chose to exclude the occipital group from our meta-analysis. 2. In analyzing the Brunoni et al. 2013 trial $^{27}$, we separated the data from participants who received sertraline and those who received placebo medication - this created two separate effects (hence although we included 10 RCTs, we analyzed $k=11$ effects).3. Segrave et al (2014) ${ }^{28}$ randomised participants to 3 groups: 1.tDCS + Cognitive Control Training (CCT), or 2.sham tDCS + CCT, or 3. tDCS + sham CCT - For the purpose of this meta-analysis, we included only 2 of the 3 study arms - comparing tDCS+CCT vs. sham tDCS+CCT. 4. Several studies reported primary outcomes at endpoints which included a follow-up period after the 
end of active treatment course. For the sake of consistency, we analysed all available data at the point when active blinded treatment ceased.

\subsubsection{Fregni $2006^{29}$.}

Study. A randomised double blind sham controlled trial in which 18 outpatients with (unipolar) MDD who had not been prescribed antidepressant medication for at least three months prior to inclusion were randomised to 2 treatment arms: either active tDCS, in which the anodal electrode was placed over F3 (10-20 International EEG System), and the cathode over the right supra-orbital area, a current of $1 \mathrm{~mA}$ being applied for 20 minutes on 5 alternate days; or sham tDCS with similar settings, but the stimulator being switched off after 5 seconds. Outcome measures involved a battery of neuropsychological rating scales; mood was assessed with the HDRS. The active treatment group showed a significantly greater improvement in mood, mean Hamilton Depression Rating Scale (HDRS) scores reduced by $58.5 \%(+/-20.4 \%)$ vs. $13.1 \%(+/-23.4 \%)$ in the sham tDCS group $[F(1,16)=19.2$, $p<0.001]$. tDCS was generally well tolerated, and no complications were reported. The active tDCS group improved on all cognitive tests, compared to their baseline scores. There was no significant cognitive improvement in the sham tDCS group. Improvement in cognitive function in the treatment group was not correlated with the improvement in mood, suggesting that cognitive and affective changes were mediated by different mechanisms.

Comment. Although involving a small number of participants, this study found evidence for antidepressant efficacy and tolerability of tDCS in MDD. The sample baseline characteristics were well defined, with moderate-tomarked baseline depressive severity (mean HDRS scores of $23.56+/-5.03$ in the active tDCS group and 25.89+/4.26 in the sham group), significant number of patients had recurrent or persistent illness.

\subsubsection{Boggio $2008^{26}$.}

Study. Following this pilot study, the same group conducted a parallel-group, double-blind, initial clinical trial of tDCS in patients with unipolar depression who had not been prescribed antidepressants for at least two months prior to trial entry. Exclusion criteria included neurological disorders, comorbid Axis I disorders, substance abuse within three months of study participation, psychotic features, bipolar disorder and Axis II disorders. Forty patients were randomized into three treatment arms (using a 2:1:1 randomization strategy): active treatment, with anodal tDCS over the left dorsolateral prefrontal cortex (DLPFC) - anode over F3 on the 10-20 International EEG System, cathode over right supraorbital region $(N=21)$; active control, with anodal tDCS of the occipital cortex anode $2 \mathrm{~cm}$ above Inion on the midline, cathode over right supraorbital region $(N=9)$; or sham control with sham 
tDCS over left DLPFC, and cathode over the right supraorbital region, the stimulator being active for an initial 30 seconds only, with ramp-up and ramp-down to mask shamming $(N=10)$. Patients received 10 sessions of tDCS on consecutive working days (no treatment being given at weekends), the current was set at $2 \mathrm{~mA}$ for 20 minutes in each session. The primary outcome measure was the 21-item HDRS, the BDI being the secondary outcome measure. Patients were rated at baseline, at end of treatment and at 15 and 30 days after treatment. tDCS was well tolerated, reported adverse effects being mild (headache, itching or redness at electrode site) and not significantly associated with group assignment. At the end of treatment, there was a significant difference between the active treatment group and the active control group $(p=0.009)$ and the sham control group $(p=0.0018)$ in HDRS score; but the control groups did not separate from each other $(p=0.6)$. The active group maintained separation from the sham control at the 30-day follow-up ( $p=0.04)$. BDI outcomes showed a similar pattern, with the active group separating from the sham group at the end of treatment $(p=0.0045$, effect size (Cohen's $d)=1.11$ ), and at 30-day follow-up ( $p=0.03$ ). There were 8 responders (HDRS scores reduced by at least $50 \%$ from baseline) in the active treatment group, compared with 2 in the active control and none in the sham control groups $(p=0.019)$. There were 5 patients in remission (HDRS $<8)$ in the active treatment group, but none in the other two groups $(p=0.02)$.

Comment. This study was larger than previous studies of tDCS in depression and demonstrated both acute efficacy and an effect lasting 30 days after treatment was stopped. The findings provide support for the use of left DLPFC anodal stimulation. This was the first study to indicate that the therapeutic effect in depression is related to the anodal effect at left DLPFC rather than to the cathodal effect at the right supra-orbital area. The tolerability of active tDCS was not significantly different to sham stimulation.

\subsubsection{Loo (2010) ${ }^{30}$}

This double-blind, sham-controlled trial of left prefrontal tDCS in depression involved 40 outpatients with DSM-IV MDD with baseline MADRS (Montgomery-Åsberg Depression Rating Scale) score of 20 or more. Exclusion criteria included diagnosis of bipolar disorder, drug or alcohol dependence or abuse, other Axis-I disorders, and neurological disorders; or the failure to respond to ECT during the index episode. Subjects were either medication-free, or had continued on the antidepressant drug to which they had not previously responded at a stable dose, unaltered for at least 4 weeks prior to study enrolment (some patients were prescribed antipsychotic drugs, and 1 patient was prescribed lamotrigine: none were prescribed benzodiazepines). Subjects were randomised to: [i] active tDCS with anodal tDCS over left DLPFC (pF3 on the10/20 EEG International System), the cathode being placed over the right lateral orbital area: $1 \mathrm{~mA}$ of current was used for 20 minutes, with 30 seconds 
of ramping-up at onset; [ii] sham tDCS with a similar montage, the current being ramped down over 30 seconds immediately after initial ramping up. Subjects in both arms underwent treatment three times weekly for five treatment sessions. All subjects then received active tDCS for another five sessions (at the same frequency). After 10 sessions, the blind was broken and patients who had received sham tDCS in sessions 1-5 were then offered another 5 sessions of active tDCS - bringing the total number of active tDCS sessions offered to all participants (regardless of treatment arm assignation) to 10. The primary mood outcome measure was the MADRS, and secondary outcome measures included the 17-item HDRS and Clinical Global Impression of Severity (CGI-S), the patient-rated BDI and Patient Global Impression Scale of Improvement (PGI-I). Subjects were assessed at baseline, after sessions 5, 10 and 15, and at 1-week and 1-month follow-up. Over the sham-controlled phase (sessions 15) there were no significant between-group differences in mood outcomes ( $p=0.87$ for MADRS). There were statistically significant differences on all measures comparing baseline mood to mood after session 10 , but no significant differences between groups. There were 6 responders (MADRS score reductions of at least 50\%) and 5 remitters (MADRS $<11$ ) in the active tDCS group, compared to 4 responders and 3 remitters in the sham group. One patient in the sham tDCS group committed suicide on the day following his first active tDCS session: the authors felt this was unlikely to have been related to the treatment this patient received (the patient had been noted to have suicidal thoughts for some months prior to his death, the suicide occurred on the first instance when he was briefly left on his own, and there were no emotional or clinical changes noted following the session of active tDCS that he had received). Adverse effects reported by the active tDCS group included redness, itchiness and tingling at electrode (mainly anode) sites; mild headaches, lightheadedness and ringing in the ears; visual changes including blurring, brighter/illuminated vision; and mild euphoria, transient hypomania ( $N=1)$, nausea, insomnia and anxiety. After session 10, subjects were asked to indicate whether they thought they had received active or sham tDCS, there being no significant in the accuracy of identification between the two groups.

Comment. This study used similar stimulation parameters to those used by Fregni et al $2006^{29,31}$ (five 20 minute sessions at $1 \mathrm{~mA}$ on alternate days with similar electrode montage,) but did not show a statistically significant separation between active and sham tDCS groups. The efficacy of active treatment over 10 sessions was comparable to that demonstrated by Boggio et al. ${ }^{26}$ who used tDCS at higher intensity (2mA, 5 times weekly for 10 sessions). The main difference between this study and the earlier trials was the greater degree of improvement in the sham tDCS group. Factors which may have contributed to this difference included the participation of patients taking antidepressants and patients with co-morbid Axis II (personality) disorders. The degree of treatment resistance in this cohort was only 'moderate' (mainly stage 0 -III on the Thase \& Rush system ${ }^{32}$, which may explain 


\subsubsection{Palm (2011) ${ }^{33}$}

This randomized double-blind placebo-controlled cross-over trial of tDCS in treatment resistant depression included 22 outpatients (14 women, 8 men: mean age 57 years): 20 with unipolar depression (17 with recurrent depression, and 3 with first-episode depression), and 2 patients with bipolar depression. All participants had failed to respond to at least two trials of antidepressants from different classes. Antidepressant medication was kept unchanged for at least 3 weeks before starting tDCS, and no medication changes were made during the study. Patients were randomized to two arms: [i] 10 sessions of active tDCS followed by 10 sessions of sham tDCS; [ii] 10 sessions of sham tDCS followed by 10 sessions of active tDCS. The anode was placed over left DLPFC (F3 on the 10-20 EEG international system), and cathode over right supraorbital region. The first 10 patients received stimulation sessions of 20 minutes at $1 \mathrm{~mA}$; the current setting was increased to $2 \mathrm{~mA}$ for the subsequent 12 patients. All patients received 20 tDCS treatments over 4 weeks. The sham stimulation included 15 -second rampup and ramp-down periods to simulate active tDCS sensations. Electrodes were soaked in tap-water for the initial 15 participants, but this was changed to normal saline solution, due to skin lesions at stimulation sites. The primary outcome measure was the HAMD-24 at 2 weeks and 4 weeks, secondary outcome measures included the BDI, CGI, PANAS and a series of cognitive tests. Twenty patients completed the trial: no significant differences were found between active and sham tDCS on the primary outcome measure - although modeling the HAMD course via mixed model analysis showed that active tDCS was superior to sham tDCS in weeks 1 and $2(p=0.0492)$. Subjective mood ratings showed a significant advantage for active over sham tDCS in positive emotions on the PANAS-pos and a trend for reduced negative emotions on the PANAS-neg. There were no significant between group differences in cognitive measures. Six of the 15 patients undergoing treatment involving use of tap-water soaked electrodes developed crusty skin lesions at the cathode site, but after normal saline solution was substituted, no further lesions were reported. Other adverse effects reported were minor, including slight headache and skin itchiness during treatment. Blinding integrity was not significantly different between groups. 
Comment. The failure of active tDCS to separate from sham tDCS in this study may have been influenced by the small sample size, the change in treatment (from $1 \mathrm{~mA}$ to $2 \mathrm{~mA}$ ) during the study, the cross-over design without an intervening no-treatment period, the higher degree of treatment resistance (average failed antidepressant trials 2.9 vs. $1.0-2.6$ in previous trials), the older age of patients (56 years, compared to $46-54$ years in previous studies), and the use of concomitant antidepressant medication.

\subsubsection{Loo (2012) ${ }^{34}$}

This randomised sham-controlled trial included 64 outpatients with DSM-IV defined major depressive episode (MDE) in the context of both unipolar and bipolar depression, with baseline MADRS score of 20 or more. Exclusion criteria included the presence of other Axis I mental disorders, excessive alcohol/drug use, neurological disorders, metal implants, history of heart or neurological disease, failure to respond to ECT in the index episode, pregnancy, and treatment with medications known to modulate tDCS effects (including benzodiazepines, anticonvulsants, dextromethorphan and pseudoephedrine). Subjects were either antidepressant medication-free for the duration of the trial, or continued on the antidepressants to which they had previously failed to respond (with no dose changes for at least 4 weeks before starting tDCS). Subjects were randomized to receive either active tDCS at $2 \mathrm{~mA}$ for 20 minutes, with ramp-up and ramp-down for 30 seconds, or sham tDCS at 1mA for 30 seconds, with rampup and ramp-down for 10 seconds. The anode was placed over left DLPFC (pF3 on the 10-20 EEG international system), the cathode lateral to the right orbit (F8 on the 10-20 EEG international system). Treatments were carried out five days per week for three weeks; each subject subsequently being offered another 15 sessions of openlabel active tDCS. Treatment responders (i.e. those whose MADRS scores reduced by at least $50 \%$ from baseline) were offered further weekly sessions of tDCS during the 1-month follow-up period. The primary outcome measure was the MADRS, assessed at baseline, after sessions 8, 15, 23 and 30, and at weeks 1 and 4 after trial completion. Other measures included the IDS, CGI-S, QIDS-C, QIDS-SR, CORE and a set of neuro-cognitive assessment tools. A significant interaction between group and time was seen on the primary outcome measure (MADRS scores), active tDCS being associated with lower MADRS scores during the sham-controlled phase ( $p=0.04$; effect size 0.49 ), but there was no significant separation on other mood outcome measures. At 1-week follow-up, 16 out of 26 subjects in the active tDCS group met criteria for response (reduction in MADRS of at least $50 \%$ from baseline) compared to 6 out of 26 in the sham tDCS group. The NNT for response at 3 weeks of active vs. Sham tDCS was 16.7; the NNT for response at 6 weeks of active tDCS vs. 3 weeks of sham tDCS was 2.6. There was one reported case of transient hypomania in the open phase of the trial (the patient had bipolar depression), other adverse effects were transient and mild to moderate in intensity, including skin redness, burning sensation, tingling and itchiness at 
electrode sites, headache, dizziness and nausea. A blinding integrity test found no significant differences between groups.

Comment. This trial included more participants and employed more robust treatment parameters (in terms of number of sessions, duration of blinded treatment, current settings, and follow-up period) than previous tDCS trials. Using the a priori primary outcome measure, this study confirmed that active tDCS has significantly greater antidepressant effects than sham tDCS over the 3-week sham-controlled phase. The magnitude of antidepressant effects (28\% reduction in MADRS) was lower than in some previous studies ${ }^{26,31}$ but patients in those trials were medication-free and the scope for tDCS-related improvement may have been greater in the absence of medication: a meta-analysis of treatment with rTMS with and without concomitant medication showed a similar finding ${ }^{35}$. The number of responders after six weeks of treatment was superior to the responder rate reported in the STAR*D trial $(28.5 \%)^{4}$. The authors suggest that extending the treatment duration to 6 weeks may be associated with additional gains (though it is unclear whether the benefit is associated with a higher number of treatments, or with the extended duration of treatment). The study findings suggest a broadly equivalent effect size to that observed with antidepressant medication, despite selection of patients with a moderate degree of treatment resistance, many of whom were already taking antidepressant medication. The results suggest that more robust tDCS treatment parameters, in terms of current, number of sessions and overall duration of treatments, may be associated with better outcomes in depression.

\subsubsection{Blumberger (2012) ${ }^{36}$}

This study explored the potential utility of tDCS in treatment-resistant depression. A total of 24 outpatients with a diagnosis of DSM-IV defined major depressive episode (MDE), a baseline HDRS score greater than 20, and fulfilling Stage II criteria (or above) on the Thase Scale for treatment resistance (i.e. failure to achieve remission or tolerate at least two trials of antidepressants from different classes) were recruited. Adjuvant medication (including antidepressants, antipsychotics and benzodiazepines) was permitted, providing dosage was stable for at least 4 weeks before study treatment started and throughout the duration of the trial. Exclusion criteria included treatment with anticonvulsants, DSM-IV substance use disorder in the 6 months prior to potential trial commencement, an unstable co-morbid medical condition, a history of seizures, pregnancy, and DSM-IV borderline or antisocial personality disorder. Subjects were randomised to receive active $(N=13)$ or sham $(N=11)$ tDCS. Treating clinicians were aware of treatment allocation, but patients and outcome assessors were blind to 
allocation. Fifteen treatment sessions were delivered on consecutive working days, over 3 weeks. The anode was placed over the left DLPFC (F3 according to the 10-20 EEG system), the cathode over the right DLPFC (F4 according to the 10-20 EEG system). Active treatment was delivered at $2 \mathrm{~mA}$ for 20 minutes, the sham treatment included an initial 30 second period of stimulation at $2 \mathrm{~mA}$, and the current was then turned off. The primary outcome measure was change in HDRS from baseline to endpoint, secondary outcome measures including the proportion achieving symptom remission $(\mathrm{HDRS}<8)$ or response to treatment $(50 \%$ reduction in severity). There was no significant difference between active and sham tDCS in the change in HDRS score $(p=0.80)$ : none of the subjects met HDRS remission criteria, and only 1 subject in each group met response criteria; and there were no significant betweengroup differences in MADRS change or in BDI-Il change. No serious adverse events were reported during the trial, though four subjects in each group reported skin tingling. Headache was reported by three subjects in the active group and by none of the sham group subjects: 1 participant in the sham group withdrew due to scalp irritation. The majority (73.7\%) of subjects correctly guessed their treatment allocation at trial endpoint (60\% in active group and $88.9 \%$ in the sham group).

Comment. This sample had a higher degree of antidepressant treatment resistance when compared to participants in previous tDCS trials (the mean number of failed antidepressant trials was larger than $4,46.2 \%$ of the active group and $18.2 \%$ of the sham group had a history of treatment with ECT; $23 \%$ of the active group and $9.1 \%$ of the sham group had failed a trial of ECT in the current episode of depression). This was the first study to focus on use of tDCS in treatment resistant unipolar depression in patients taking a wide variety of concomitant medication. The study limitations include small sample size, and probable under-powering (the power calculation required 48 subjects, but only 24 were recruited): recruitment was stopped on ethical grounds after an interim analysis found no difference between treatment groups. The degree of treatment resistance in this cohort may have been too great to permit a detectable effect; and blinding may have been sub-optimal. Subjects who started antidepressants four weeks prior to the trial may have experienced antidepressant drug-related treatment effects during the trial. The active stimulation group was more treatment-resistant and more were taking benzodiazepines (which may impair the effects of neurostimulation).

\subsubsection{Brunoni (2013) ${ }^{27}$}

This study explored the comparative safety and efficacy of tDCS, the antidepressant sertraline, and placebo, as well as their combinations in the treatment of Major Depressive Disorder. A total of 120 subjects with DSM-IV defined major depressive disorder (MDD), a baseline $\mathrm{HDRS}_{17}$ score greater than 17 , and a low suicide risk, took 
part in this double-blind randomized controlled trial. All subjects were free of antidepressant, antipsychotic and anticonvulsant medication for at least 5 half-lives of the drug, before onset of trial. Adjuvant benzodiazepines were permitted. Exclusion criteria included: other axis-I disorders (co-morbid anxiety disorders were permitted), substance use disorders, axis-II disorders, previous neurological conditions, severe axis-III disorders and specific contra-indications to tDCS (e.g. metallic implants in the head). Subjects who were prescribed sertraline in the current depressive episode were excluded. Subjects were randomized to one of four groups: Active tDCS + sertraline, tDCS + placebo medication, sham tDCS + sertraline, sham tDCS + placebo medication. Participants and assessors were blinded to treatment allocation; the treating clinicians were aware of allocation, but their interaction with participants was kept to a minimum. Medication and tDCS were initiated concurrently. tDCS was delivered using a bi-frontal montage, with the anode placed over the Left DLPFC (F3 according to the 10-20 EEG system), and the cathode over Right DLPFC (F4 according to the 10-20 EEG system). Twelve tDCS sessions were delivered - 10 sessions on consecutive week days (Monday to Friday) and two subsequent sessions at fortnightly intervals. Active tDCS was delivered using a current setting of $2 \mathrm{~mA}$, for 30 minutes per session; sham tDCS was delivered by switching the stimulator off after 1 minute. Sertraline was administered at a fixed dose of $50 \mathrm{mg}$ per day. The primary outcome measure was change in MADRS score at 6 weeks; secondary outcomes were clinical response (defined as $>50 \%$ reduction of the baseline MADRS score), clinical remission (defined as a MADRS score $\leq 10$ ), and scores on the HDRS17, Global Impression-Severity of Illness scale, and Beck Depression Inventory). At the main end point (6 weeks), there was a significant difference in MADRS scores when comparing the combined treatment group (sertraline/active tDCS) vs. sertraline + sham tDCS (mean difference, 8.5 points; $95 \% \mathrm{Cl}, 2.96$ to 14.03; $p=.002$ ), tDCS + placebo (mean difference, 5.9 points; $95 \% \mathrm{Cl}, 0.36$ to $11.43 ; p=.03$ ), and placebo/sham tDCS (mean difference, 11.5 points; $95 \% \mathrm{Cl}, 6.03$ to $17.10 ; p_{-} .001$ ). Analysis of tDCS + placebo medication vs. sertraline + sham tDCS demonstrated comparable efficacies (mean difference, 2.6 points; $95 \%$ Cl, _2.90 to 8.13; $p=.35$ ). Use of tDCS + placebo medication (but not sertraline + sham tDCS) was superior to placebo + sham tDCS. Common adverse effects did not differ between interventions, except for skin redness on the scalp in active tDCS $(p=.03)$.

Active vs. sham tDCS was significantly superior for all outcomes (Odds Ratios for response and remission were, respectively, $1.63 ; 95 \% \mathrm{Cl}=1.26-2.12$ and $2.50 ; 95 \% \mathrm{Cl}=1.26-2.49)$. There were 7 episodes of treatmentemergent mania or hypomania, five of which occurred in the combined treatment group.

\section{Comment.}

This is largest trial to date of tDCS in depression; the factorial (2X2) design enables the authors to address issues of monotherapy vs. co-initiation of tDCS and antidepressant medication. The combination of sertraline and tDCS 
(initiated simultaneously) was associated with better outcomes than in other arms of the trial, this may be a pointer to particular efficacy of co-initiated combination therapy. The choice of primary outcome endpoint at 6 weeks differs from all previous tDCS RCTs in depression, as it encompasses both the 2 week intensive treatment phase, and 2 fortnightly add-on tDCS sessions.

\subsubsection{Bennabi (2014) 37}

This study examined the utility of tDCS in treatment resistant depression. A total of 24 patients meeting diagnostic criteria for DSM-IV criteria for Major Depressive Disorder, with a baseline MADRS score greater than 24, and meeting stage II criteria (or above) for treatment resistance. All patients received a constant dose of escitalopram $(10-20 \mathrm{mg} / \mathrm{d})$ over 4 weeks prior to tDCS treatment initiation. Concomitant treatment with benzodiazepine and/or second generation antipsychotics was allowed. Exclusion criteria included bipolar depression, psychotic features, neurological/ severe organic disease or treatment with First Generation Antipsychotics. Subjects were randomised to receive active $(N=12)$ or sham $(N=12)$ tDCS. Both subjects and clinicians were blinded to allocation. Ten treatment sessions were delivered over five consecutive days (two treatments per day). The anode was placed over the Left DLPFC, and the cathode over the Right supraorbital area. Active tDCS was delivered using $2 \mathrm{~mA}$ intensity for 30 minutes per session. Sham tDCS was delivered using identical settings, but the current was gradually ramped down to zero mA. Depression severity was assessed using the HDRS21 (primary outcome measure), MADRS, and BDI. Response was defined as a decrease of at least 50\% from baseline HDRS score, remission was defined as a HDRS score of 8 or less. There was no significant difference between active and sham tDCS in the change in HDRS score $(p=0.69)$ : immediately after the course ended, in the active tDCS group there were 3 subjects who responded and 2 who met criteria for remission. In the sham tDCS group there was 1 responder and no remitters. The authors comment that one subject developed mania and withdrew from the study, but they do not mention the group allocation for this subject. There were no other serious adverse events. The authors do not supply information about the degree of blinding integrity.

Comment. This study is limited by the small number of subjects. There is considerable level of treatment resistance, and similarly to the Blumberger study ${ }^{36}$, subjects started a new antidepressant 4 weeks prior to the trial, and may have experienced medication-related effects.

\subsubsection{Segrave $(2014)^{28}$}


This was the first study to examine the use of tDCS in conjunction with Cognitive Control Training (CCT), a type of neurocognitive training, which like tDCS is aimed at activating the DLPFC. The rationale for combining both modalities is that there is evidence to suggest that there are more pronounced functional outcomes when tDCS is applied to active brain regions, rather than to areas at rest. ${ }^{38-40}$. CCT is a novel therapeutic modality for depression, aimed at activation of the DLPFC through two targeted cognitive activities designed in reference to functional imaging data, to activate the DLPFC ${ }^{28}$ : the first, a modified Wells Attentional Training (WAT) paradigm is a guided auditory process, directing attention through phases of focused attention, switching attention and divided attention; the second, is a modified Paced Serial Addition Task (PASAT), a mental arithmetic task. 27 subjects with DSM-IV Major Depressive Episode, whose baseline MADRS score was greater than 18 took part in this study. Exclusion criteria included: Lifetime history of neurological illness, mania, hypomania PTSD or psychosis, and substance use disorders in the year prior to study initiation. Subjects were either not prescribed antidepressant medication, or were stable on the same dose for at least 4 weeks prior to study initiation. Subjects were randomised to receive five sessions of either: 1.tDCS + CCT $(N=9)$, or 2.sham tDCS + CCT $(N=9)$, or 3. tDCS + sham $\mathrm{CCT}(N=9)$. Participants and raters were blinded to allocation, but the operator was aware of allocation. Sessions were delivered on 5 consecutive working days. Active tDCS was delivered for 24 minutes per session using current setting of $2.0 \mathrm{~mA}$. Sham tDCS was delivered using a 2 minute fade out period. CCT started 2 minutes after initiation of tDCS session. The anode was placed over Left DLPFC (F3 according to the 10-20 EEG system), the cathode over the lateral aspect of the Right orbit (F8 according to the 10-20 EEG system). Results: there were no drop-outs during the course of treatment, tDCS was well tolerated and no serious adverse events were reported. There was a significant difference between the three arms in respect of the change in MADRS scores over time $\mathrm{F}_{(4,48)}=4.63(p=0.03)$. Post-hoc analysis demonstrates significant reduction in MADRS scores for subjects in the sham-tDCS+CCT group $(p=0.02)$, and in the tDCS+sham-CCT group $(p=0.04)$; there was a trend towards significance in the tDCS+CCT group $(p=0.06)$. At 3-week follow-up, only the tDCS+CCT group showed significant difference from baseline MADRS scores $(p<0.001)$. There were no significant differences in response rates immediately following the 5 treatment course $(p=0.08)$. At 3 week follow up, there was a significant difference in response rates $(p=0.04)$ : $\mathrm{tDCS}+\mathrm{CCT} 44 \%$, sham tDCS+CCT $11 \%$, tDCS +sham CCT $0 \%$. For the purpose of this meta-analysis, we included only 2 of the 3 study arms - comparing tDCS+CCT vs. sham tDCS+CCT.

Comment. An interesting finding was the delayed onset of maximal therapeutic efficacy of the tDCS+CCT combination. This is not consistent with a previous study of the tDCS+CCT combination in healthy individuals ${ }^{41}$. This finding supports the inclusion of follow-up phases in future tDCS studies. 


\subsubsection{Brunoni $(2014)^{42}$}

This is the second study examining the combination of tDCS and CCT for the treatment of depression. Subjects fulfilled criteria for DSM-IV major depressive episode, with a baseline HDRS score $>21$; the age range was 18-65. Exclusion criteria included: 1 . Bipolar disorder, substance use disorders, schizophrenia, personality disorders, and other co-morbid psychiatric disorders apart from anxiety disorders. 2. Neurological conditions. 3. Patient prescribed antipsychotics or tricyclic antidepressants. All subjects were taking antidepressant medication (SSRI or SNRI) with no dose changes for at least six weeks prior to Study initiation. Subject were randomised to 10 treatments on consecutive working days, consisting of either: 1 . CCT+sham tDCS $(n=17)$ or 2. CCT+tDCS $(N=20)$. The primary endpoint was 4 weeks post initiation ( 2 weeks of active treatment and 2 weeks of follow-up). tDCS parameters used were: 30 minutes per session at $2 \mathrm{~mA}$ current setting, Anode over Left DLPFC (F3 according to the 10-20 EEG system), Cathode over Right DLPFC (F4 according to the 10-20 EEG system). Sham tDCS used 30 sec ramp-in, $30 \mathrm{sec}$ active stimulation and $15 \mathrm{sec}$ ramp-out. The CCT intervention included only the modified PASAT, and was delivered during the final 15 minutes of each tDCS session. Results; tDCS was well-tolerated and no adverse effects were reported. There was 1 drop out from the active tDCS+CCT and 3 drop outs from the sham tDCS $+C C T$ groups during the active treatment phase. There were no statistically significant differences between the groups in respect of the primary outcome measure. Both groups demonstrated similar reductions of HDRS scores at week $2(p=0.91)$ and at week $4(p=0.71)$. There were no statistically significant differences in categorical response and remission rates at week 2 or at week 4 . Older subjects demonstrated a stronger additional effect of tDCS when combined with CCT. There were no additional improvement in depressive symptoms during the 2-week follow-up period (in contrast to the findings in the previous CCT+tDCS study ${ }^{28}$.

Comment. This study demonstrated no statistically significant differences between active vs. sham tDCS, when added to a course of CCT in subjects with unipolar depression. The lack of significant difference at the end of the active treatment phase is consistent with the previous CCT+tDCS study ${ }^{28}$; however, in this study there was also no difference after a 2 week follow-up period.

\subsection{Published meta-analyses}

Our search of the literature identified 3 published meta-analyses of tDCS in depression. Their main design features and those of our own meta-analysis are summarised in table 2. 


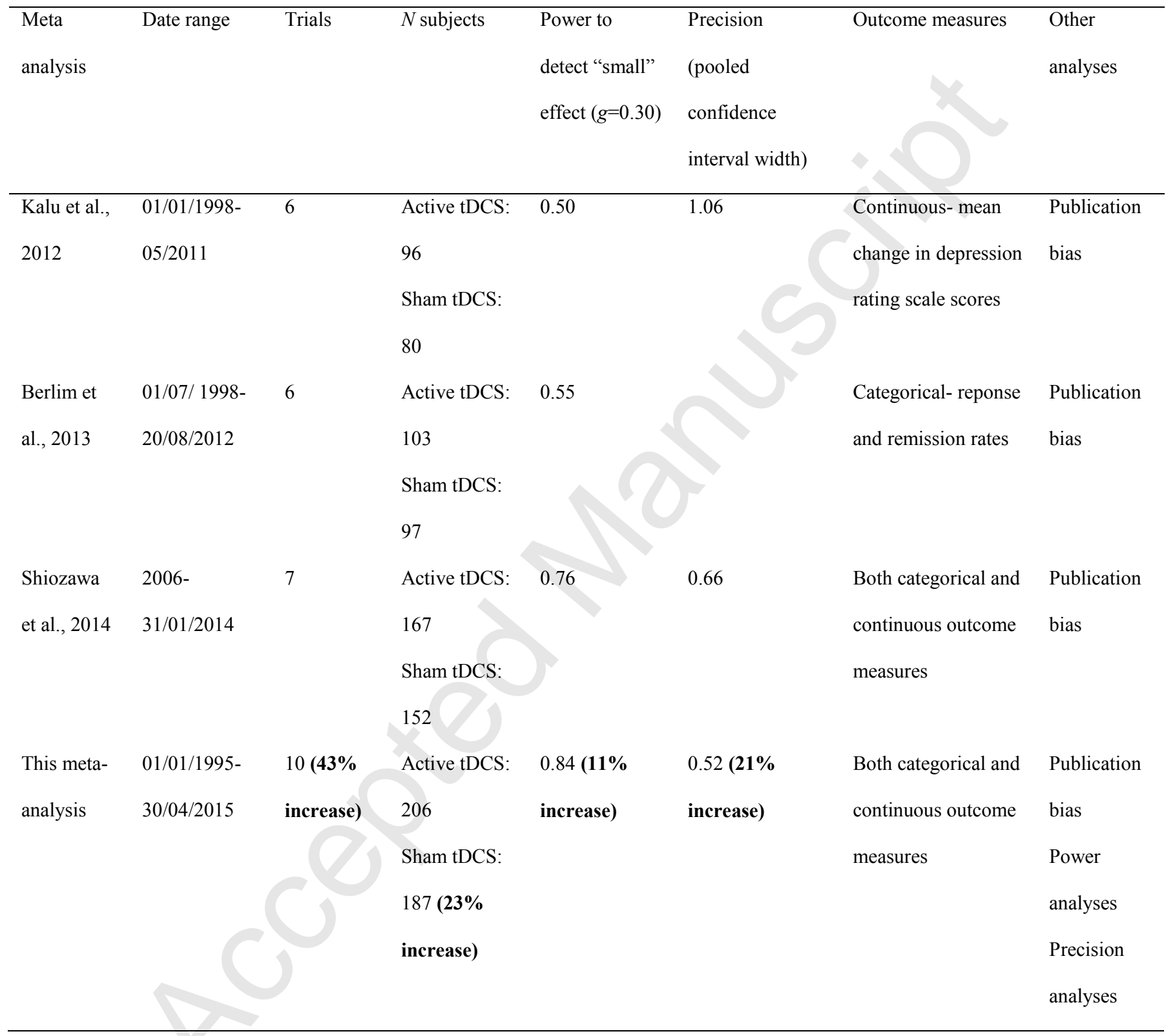

Table 2: Comparison of previously published and current meta-analyses of tDCS in depression 
3 The first systematic review and meta-analysis of tDCS in the treatment of depression included randomized parallel

4 or cross-over studies of active tDCS vs. sham tDCS controls, double blind allocation to treatment, and outcome measures including a clinician-rated depressive symptom severity scale (HDRS or MADRS), and an account of the change (\%) in symptom severity. Six randomized controlled trials including a total of 96 patients in active tDCS arms and 80 patients in sham tDCS arms were included ${ }^{26,29-31,33,34}$. Depressive symptom severity was reduced by a weighted mean of $28.9 \%$ (14.6-60\%). A weighted mean of $21.8 \%$ (range: $0-80 \%$ ) of RCT participants receiving active tDCS experienced categorical response (50\% symptom severity reduction), and $6.1 \%$ (range $0-23 \%$ ) experienced symptomatic remission (HDRS score $<8$ or MADRS score $<11$ ). The wide variability between studies may have reflected differing levels of treatment resistance, concomitant medication use, and variability in delivery of tDCS between trials. The pooled estimate of effect size (Hedges' $g$ ) for depressive severity reduction between active and sham tDCS was $0.74(Z=2.76, p=0.006,95 \%$ confidence interval $0.21-1.27)$. All four studies which included 1-month follow-up data reported that the reduction in symptom severity with treatment was maintained (and in 2 studies increased in magnitude) at follow-up. No significant correlations were found with baseline symptom severity, concomitant antidepressant use, stimulation current strength or total number of sessions. The most common adverse effects in both active and sham tDCS study-arms were headaches, and local itchiness and/or redness at electrode sites. Skin-lesions were associated with the use of tap-water instead of saline solution for electrode preparation. Four cases of tDCS-associated hypomania were reported - 3 in published trials ${ }^{30,34,43}$ and one in an ongoing trial ${ }^{44}$. The authors conclude that tDCS is a potentially effective treatment for depression, but acknowledge limitations such as the small number of available studies, small number of participants, and heterogeneity in participant populations and treatment parameters. The authors called for large-scale studies with longer follow-up periods in more representative participant groups.

\subsubsection{Berlim et al (2013) meta analysis ${ }^{13}$}

The second meta-analysis of tDCS in the treatment of MDD investigated the utility of tDCS using response and remission rates as outcome measures. Systematic review searched for publications which fulfilled the following inclusion criteria: randomised, sham-controlled, double-blind, parallel or cross-over design with at least 5 subjects randomised to each study arm; participants aged 18-75, with a primary diagnosis of DSM-IV or ICD-10 Major Depressive Episode (MDE) of a unipolar or bipolar nature; treated with at least 5 sessions of tDCS, at an intensity of at least $1 \mathrm{~mA}$, with the anode over the left DLPFC, tDCS being administered as either monotherapy or 
augmentation strategy; publications in the English language. Exclusion criteria included enrolment of subjects with 33 subsets of depression (e.g. post-partum or vascular depression); contemporaneous initiation of tDCS and another treatment for depression (e.g. antidepressant medication); and studies, which did not report response and/or remission rates. The literature search yielded 6 RCTs, including 5 of the 6 trials incorporated by Kalu et al. ${ }^{12}$ in their meta-analysis ${ }^{26,30,31,33,34}$ : together with a more recent $\mathrm{RCT}{ }^{36}$. Overall, the dataset included 200 subjects with MDE, 103 of whom were randomised to active tDCS. Subjects received a $10.8+/-3.76$ sessions of tDCS. The cohort had failed to respond to a mean of $2.36+/-1.19$ trials of antidepressants. There was no significant difference in baseline depression severity between the active and sham tDCS groups $(p=0.66)$. Using drop-out rates to assess overall treatment acceptability, there were no significant differences in drop-out rates between active (4.8\%) and sham (5.1\%) tDCS $(p=0.86)$. There was no significant difference in blinding integrity between active and sham tDCS ( $p=0.41) ; 44.1 \%$ of active tDCS participants correctly guessed treatment allocation at study end, vs. $53.7 \%$ of sham tDCS participants. The overall rates of response and remission were not significantly different between the active and sham tDCS groups: response rates were $23.2 \%$ for active tDCS vs. $12.4 \%$ for sham tDCS, pooled $O R=1.97(95 \% \mathrm{Cl}=0.85-4.56 ; p=0.11)$; and remission rates were $12.2 \%$ for active tDCS vs. $5.4 \%$ for sham tDCS, pooled $O R=2.13(95 \% \mathrm{Cl}=0.64-7.06 ; p=0.22)$. Active tDCS significantly out-performed sham tDCS when used as a monotherapy for MDE: $O R=7.54(95 \% \mathrm{Cl}=1.630-34.8 ; p=0.01)$. There was no association between number of treatment sessions ( 5 vs. 10 treatments) or electrical current used ( $1 \mathrm{vs.} 2 \mathrm{~mA}$ ) and treatment efficacy. The authors highlighted limitations, including small sample sizes, little or no follow-up after treatment protocol is ended, and no differentiation between effects in unipolar and bipolar depression.

\subsubsection{Shiozawa et al (2014) ${ }^{14}$}

53 This meta-analysis aimed to improve on previous meta-analyses by addressing two main areas: First, previous

54 meta-analyses had utilised different methodologies for calculating effect size: the Kalu meta-analysis ${ }^{12}$ used continuous depression severity scores, whereas the Berlim meta-analysis ${ }^{13}$ used categorical response/remission rates. Second, previous meta-analyses had not includes data from the biggest tDCS for depression trial to date ${ }^{27}$. The inclusion criteria were: randomised, sham-controlled trials, providing data including continuous depression scores and categorical response + remission rates. The meta-analysis includes 7 RCTs ${ }^{26,27,29,30,33,34,36},(N=259$ participants). Active tDCS significantly outperformed sham-tDCS on continuous depression scores $(g=0.37 ; \mathrm{Cl}$ 0.04-0.7). Odds Ratios for response and remission were respectively $1.63 ; 95 \% \mathrm{Cl}=1.26-2.12$ and $2.50 ; 95 \%$ $\mathrm{Cl}=1.26-2.49$. 


\section{$63 \quad$ 3.2.4 Discussion of previous meta-analyses}

64 The three published meta-analyses of tDCS for depression present inconsistent conclusions, possible due to a 65 number of factors First, choice of outcome measures for calculating effect sizes - the 2012 meta-analysis ${ }^{12}$ used 66 percentage change in depressive rating scale scores from baseline to endpoint, whereas the 2013 meta-analysis ${ }^{13}$ 67 used categorical response and/or remission rates, dichotomous outcomes which effectively raise the threshold for 68 demonstrating differential effects between active and sham interventions. The 2014 meta-analysis ${ }^{14}$ utilises both 69 approaches: its conclusions are aligned with previous findings in respect of continuous depression rating scale 70 data ${ }^{12}$, but are at odds with previous conclusions regarding response/remission rates ${ }^{13}$ - this may be due to the 71 different studies included in this meta-analysis (in particular, the inclusion of the large 2013 trial ${ }^{27}$ ). Second, the 722013 meta-analysis includes a study which recruited participants with highly treatment-resistant depression 73 (including those whose depression failed to respond to ECT, who were excluded from earlier trials). Third, there 74 was an increased proportion of studies involving tDCS augmentation vs. Monotherapy in the 2013 meta-analysis: 75 tDCS trials in which participants are allowed to continue antidepressant medication. This may limit the apparent 76 effectiveness available to be demonstrated by tDCS, as the scope for active tDCS to increase the response and/or 77 remission rates may be reduced when subjects are concurrently treated with antidepressant medication; some 78 medications (for instance anticonvulsants) may actually reduce the efficacy of tDCS; and there may be an increased 79 degree of treatment refractoriness in patients recruited to these trials. The 2014 meta-analysis ${ }^{14}$ included large $80 \mathrm{RCT}^{27}$ in which ADM and tDCS were co-initiated - this group outperformed other arms of the trial (as well as 81 giving rise to most manic switches), which may indicate a particular increase in antidepressant efficacy when tDCS $82 \&$ Sertraline are co-initiated.

91 Factors associated with these differential outcomes can be divided into participant-related factors and tDCS92 related factors (table 3) 
94 Participant-related factors include treatment-resistance level, and concurrent treatment with medication or with

95 CCT. Using the mean number of antidepressant medication trials before starting tDCS as a measure of the mean 96 level of treatment resistance in each cohort, it seems that 4 RCTs ${ }^{28,33,36,37}$ recruited patients with higher levels of 97 treatment resistance. The mean number of failed ADM trials in these studies was greater than 2 (compared to 98 1.0-1.7 in all other RCTs for which data was available). All these studies found no advantage of active over sham 99 tDCS, whereas 3: out of 4 RCTs in which tDCS was used for less treatment-resistant depression found evidence for superiority of active over sham tDCS ${ }^{26,27,34}$. The available data suggest that active tDCS is more likely to outperform sham tDCS in patients who have failed less than two trials of ADM, than in those who have failed more than two trials of ADM. It is notable that a similar pattern is seen in antidepressant drug RCTs: compared to patients who have not been treated or who have failed one ADM trial, patients who have not responded to more than 2 trials of ADM demonstrate a significantly lower response rate to subsequent ADM trials ${ }^{45}$. As regards concurrent medication usage, 2 out of 10 RCTs did not permit concurrent ADM use during the trial, both found significant superiority of active over sham tDCS ${ }^{26,29,42}$. The potential for additional improvement with tDCS in patients who are already prescribed an antidepressant or undergoing CCT may be limited, in comparison with patients who are receiving tDCS monotherapy. Antidepressants may also directly interfere with tDCS efficacy.

TDCS-related factors include current settings, number of tDCS sessions and session duration, as well as electrode placement. Of the 4 positive RCTs, three used the higher $(2 \mathrm{~mA})$ rather than the lower $(1 \mathrm{~mA})$ current setting. By contrast, of the six failed RCTs, two used the lower current setting (1mA) in all participants ${ }^{30}$, or in some of them ${ }^{33}$. Palm et al. ${ }^{33}$ compared the outcomes for participants who received tDCS at $1 \mathrm{~mA}$ vs. $2 \mathrm{~mA}$ and found there was no significant difference $(p=0.38)$ between groups: however, this trial recruited participants with treatment resistant depression, in whom the difference in efficacy between current levels may not have been sufficiently great to be associated with significantly different treatment outcomes. As regards the number of sessions, the number of sessions used in RCTs ranges from 5 to 15 . Of the four positive RCTs, one involved 5 treatment sessions, two involved 10 treatment sessions, and a single trial involved 15 sessions. The negative RCTs include 2 trials involving 5 sessions, three involving10 sessions, and one involving 15 sessions.

Of course, it may be that these factors influence the efficacy of tDCS in an additive fashion: among the 6 failed RCTs, 4 trials recruited participants with higher levels of treatment-resistance. The only failed RCT which reported recruiting participants with lower levels of treatment-resistance mean number of failed ADM courses <2) ${ }^{30}$, combined a short course of tDCS (5 treatment sessions), with a low current setting (1mA). 


\begin{tabular}{|c|c|c|c|c|c|c|c|c|c|c|c|c|c|}
\hline Name & $\begin{array}{l}\text { Diagnosi } \\
\text { s }\end{array}$ & $\begin{array}{l}\mathrm{N} \\
\text { active, } \\
\text { sham }\end{array}$ & $\begin{array}{l}\text { Age } \\
\text { (S.D.) }\end{array}$ & $\begin{array}{l}\text { Gender } \\
\% \\
\text { Female }\end{array}$ & $\begin{array}{l}\text { Rating } \\
\text { Scale }\end{array}$ & $\begin{array}{l}\text { tDCS } \\
\text { Curren } \\
t(m A)\end{array}$ & $\begin{array}{l}\text { tDCS } \\
\text { Session } \\
\text { Duration } \\
\text { (min) }\end{array}$ & $\begin{array}{l}\text { tDCS } \\
\text { Montage: } \\
\text { anode, } \\
\text { cathoode }\end{array}$ & $\begin{array}{l}\text { Number of } \\
\text { tDCS } \\
\text { Sessions }\end{array}$ & $\begin{array}{l}\text { Treatment } \\
\text { resistance } \\
\text { level }\end{array}$ & $\begin{array}{l}\text { Con- } \\
\text { current } \\
\text { ADM }\end{array}$ & $\begin{array}{l}\text { Con- } \\
\text { current } \\
\text { CCT }\end{array}$ & $\begin{array}{l}\text { Depression } \\
\text { outcome }\end{array}$ \\
\hline Fregni $2006^{29}$ & Unipolar & $\overline{9,9}$ & $48.2(10)$ & No data & MADRS & 1.0 & 20 & F3, FP2 & 5 & no data & No & No & Active>Sham \\
\hline Boggio $2008^{26}$ & Unipolar & 21,10 & $49(7.4)$ & $67.5 \%$ & HDRS & 2.0 & 20 & F3, FP2 & 10 & 1.7 & No & No & Active $>$ Sham \\
\hline Loo $2010^{30}$ & Unipolar & 20,20 & $\begin{array}{c}47.3 \\
(11.3)\end{array}$ & $55 \%$ & MADRS & 1.0 & 20 & F3 , FP2 & 5 & $\begin{array}{l}\text { Active } 1.0 \\
\text { Sham } 1.7\end{array}$ & Yes & No & Active $=$ Sham \\
\hline Palm 201133 & $\begin{array}{l}\text { Unipolar } \\
\text { \& bipolar }\end{array}$ & 11,11 & $57(12)$ & $50 \%$ & HDRS & $\begin{array}{l}1.0 \text { or } \\
2.0\end{array}$ & 20 & F3, FP2 & 10 & $\begin{array}{l}\text { Active } 2.9 \\
\text { Sham } 2.91\end{array}$ & Yes & No & Active $=$ Sham \\
\hline Loo $2012^{34}$ & $\begin{array}{l}\text { Unipolar } \\
\text { \& bipolar }\end{array}$ & 33,31 & $\begin{array}{c}48.2 \\
(12.5)\end{array}$ & $46.6 \%$ & MADRS & 2.0 & 20 & $\mathrm{~F} 3, \mathrm{~F} 8$ & 15 & $\begin{array}{l}\text { Active } 1.71 \\
\text { Sham } 1.79\end{array}$ & Yes & No & Active $>$ Sham \\
\hline $\begin{array}{l}\text { Blumberger } \\
2012^{36}\end{array}$ & Unipolar & 13,11 & $\begin{array}{c}42.7 \\
(11.6)\end{array}$ & $45.6 \%$ & HDRS & 2.0 & 20 & $\mathrm{~F} 3, \mathrm{~F} 4$ & 15 & $\begin{array}{l}\text { Active } 4.3 \\
\text { Sham } 4.1\end{array}$ & Yes & No & Active $=$ Sham \\
\hline Brunoni $2013^{27}$ & Unipolar & 30,30 & $42(12)$ & $68 \%$ & MADRS & 2.0 & 30 & F3, F4 & $10+2$ & 1.7 & Yes & No & Active $>$ Sham \\
\hline Bennabi2014 ${ }^{37}$ & Unipolar & 12,12 & $\begin{array}{c}61.8 \\
(16.3)\end{array}$ & $75 \%$ & HDRS & 2.0 & 30 & F3, FP2 & $\begin{array}{l}10 \text { ( } 5 \text { days } \\
\text { twice daily) }\end{array}$ & $100 \%>2$ & Yes & No & Active $=$ Sham \\
\hline Segrave $2014^{28}$ & Unipolar & 9,9 & $\begin{array}{c}40.4 \\
(14.5)\end{array}$ & $37 \%$ & MADRS & 2.0 & 24 & F3, F8 & 5 & 3.0 & Yes & Yes & Active $=$ Sham \\
\hline Brunoni $2014^{42}$ & Unipolar & 20,17 & $18-65$ & $70.2 \%$ & HDRS & 2.0 & 30 & F3, F4 & 10 & $35-41 \%>2$ & Yes & Yes & Active $=$ Sham \\
\hline
\end{tabular}

Table 3: Participant-related factors, tDCS-related factors and tDCS trial depression outcomes: RCTs of tDCS in depression listed in chronological order. Depression outcome is a categorical statement as to whether active tDCS out-preformed sham tDCS in terms of depression outcome measures $(p \leq 0.05)$, at the end of active treatment course. ADM = Antidepressant Medication. CCT=Cognitive Control Training. The degree of treatment resistance is indicated by the number of ADM trials failed prior to starting tDCS. 


\subsection{Analysis of published meta-analysis efficacy factors}

Consideration of the 3 published meta-analyses, provides the following efficacy-related insights:

3.4.1 Kalu et al. $2012^{12}$. This meta analysis utilised continuous outcome measures and showed that active tDCS was associated with significant reduction in symptom severity compared with sham tDCS. It found that metaregression with participant related factors (baseline severity, concurrent treatment with antidepressant medication), and with tDCS-related factors (number of sessions, current settings) did not yield any significant correlations.

3.4.2 Berlim et al. $2013^{13}$. This meta-analysis utilised categorical response and remission outcome measures, and concluded that there was insufficient evidence to support tDCS as superior to placebo in achieving response or remission from depression. Neither participant-related, nor treatment-related factors were significantly correlated with differential treatment outcomes.

3.4.3 Shiozawa et al. $2014^{14}$. This meta-analysis utilised both continuous and categorical outcome measures, and found that tDCS with statistically superior to sham tDCS in the treatment of depression In both outcome domains. Meta-regression of both categorical and continuous outcome measures did not yield any statistically significant correlation between participant-related or treatment-related factors and treatment outcomes.

\subsection{Published meta-analysis safety and tolerability findings:}

The three published meta-analyses contribute the following insights into safety and tolerability:

1. Kalu et al. $2012{ }^{12}$. The most common adverse effects reported by studies included in the systematic review were of a minor nature including headaches, itchiness and redness of skin underlying the electrodes. Skin lesions reported by Palm et al. $2011^{46}$ were not observed once the electrodes were soaked in saline solution rather than tap water. The authors note that although adverse effects were more commonly reported in the active tDCS than in sham tDCS groups, the differences were not statistically significant. There were several reports of 'treatment emergent' hypomanic episodes.

2. Berlim et al. $2013^{13}$. Dropout rates at study end did not demonstrate a statistically significant difference between active and sham tDCS groups. The authors did not comment on safety aspects. 
3. Shiozawa et al. $2014^{14}$. No statistically significant differences were found between acceptability (as measured by dropout rates) of active versus sham tDCS.

\section{CURRENT META-ANALYSIS: Continuous Treatment effects}

Across all studies the combined treatment effect was significant and consistent with a small effect size $(k=11$, $g=0.30,95 \% \mathrm{Cl}=[0.04,0.57], p=.027)($ see Figure $2 \mathrm{a})$. The 'probability of superiority' metric ${ }^{30}$ indicated a $62 \%$ chance that a randomly sampled individual receiving active tDCS would have a greater reduction in depressive symptoms than a randomly sampled individual receiving sham tDCS $95 \% \mathrm{Cl}$ [52\% 72\%]. A 'leave one out' analysis revealed that removing Boggio et al $(2008)^{26}$, Loo et al $(2012)^{34}$, or the concurrent Sertraline group of Brunoni et al $(2013)^{27}$ would reduce the effect to non-significance (largest $p=.078$, smallest $g=0.23$ ). The meta-analytic combination of effects yielded a valuable increase in precision. From the earliest study to the most recent, the margin of error (width of one arm of the confidence interval: MOE) decreased from 1.18 to 0.26 . The test for heterogeneity was significant $(Q(10)=19.27, p=.037)$ and the $I^{2}$ statistic indicated that $40 \%$ of the heterogeneity between studies could not be accounted for by sampling variability, justifying the use of a random effects model.

\subsection{Fail-safe $\mathrm{N}$ analyses}

The "fail safe $\mathrm{N}$ " calculation using the Rosenthal approach ${ }^{22}$, revealed that 27 unpublished studies averaging null results would be required in order for the treatment effect to dip below significance $(a=.05)$. Additionally, we used the Orwin fail-safe $N$ calculations ${ }^{21}$ to estimate the number of unpublished studies averaging null results that would be required to reduce the effect size to a range of target levels, this data is plotted in Figure $2 \mathrm{~b}$.

\subsection{Publication bias}

A funnel plot of the outcomes is shown in Figure 2c. To examine the sensitivity of the data to publication bias we employed the nonparametric "trim and fill" method ${ }^{47}$. The procedure estimated that one study (on the left of the summary effect) could have been suppressed due to publication bias. Imputing this missing study and repeating the analyses marginally reduced the effect size $(k=12, g=0.22,95 \% \mathrm{Cl}=[-0.11,0.56], p=.195)$.

\subsection{Precision and power analyses}




\subsection{Moderator analysis for continuous outcomes}

A summary of the one-moderator models is shown in table 4. Treatment resistance level was removed due to the inconsistent reporting (for separate groups, or for all participants combined, or no exact values) which prevented a useful and informative component of this analysis. Meta regression did not detect any significant moderators of the treatment effect - no one-moderator model provided a better fit to the data than an empty (no moderator model). In a multiple regression model, we calculated the proportional contribution of each moderator to the overall $R^{2}$, collapsed across orderings of regressors. This revealed that concurrent Antidepressant Medication (ADMs) and concurrent Cognitive Control Training (CCT) were the most important predictors, both impacted negatively on the treatment effect and accounted for $47 \%$ and $36 \%$ of the total variation explained respectively (Figure 2e). Closer examination of these factors revealed that samples who were not taking concurrent ADMs $(g=0.71,95 \% \mathrm{Cl}[0.121 .29], p=.019)$ had a larger treatment effect than those who were $(g=0.18,95 \% \mathrm{CI}[-0.16$ $0.51], p=.302)$. Similarly, samples that did not receive concurrent CCT ( $g=0.39,95 \% \mathrm{CI}[0.130 .65], p=.004)$ had a larger treatment effect than those who did ( $g=-0.20,95 \% \mathrm{Cl}[-0.820 .41], p=.517)$. No interactions between 216 moderators could be tested, due to empty cells in the model matrices. 
2

3

4217

5

6

7

8

9

10

11

12

13

14

15

50

51

52

\begin{tabular}{|c|c|c|c|c|c|c|c|}
\hline Moderator & $k$ & $d f$ & Qo & $p$ & $R^{2}$ & $b_{0}$ & $b_{1}$ \\
\hline Current & 11 & 1 & 0.50 & .481 & 0.0 & 0.57 & -0.30 \\
\hline $\begin{array}{l}\text { Number of } \\
\text { sessions }\end{array}$ & 11 & 1 & 0.00 & .964 & 0.0 & 0.28 & 0.00 \\
\hline $\begin{array}{l}\text { Session } \\
\text { length }\end{array}$ & 11 & 1 & 014 & .705 & 0.0 & 0.35 & -0.11 \\
\hline $\begin{array}{l}\text { Total tDCS } \\
\text { time }\end{array}$ & 11 & 1 & 0.07 & .791 & 0.0 & 0.43 & -0.00 \\
\hline Total current & 11 & 1 & 0.41 & .783 & 0.0 & 0.41 & -0.00 \\
\hline ISI & 11 & 1 & 0.50 & .481 & 0.0 & 0.26 & 0.30 \\
\hline Diagnosis & 11 & 1 & 0.00 & .995 & 0.0 & 0.31 & -0.00 \\
\hline $\begin{array}{l}\text { Concurrent } \\
\text { ADMs }\end{array}$ & 11 & 1 & 2.35 & .125 & 0.0 & 0.70 & -0.53 \\
\hline $\begin{array}{l}\text { Concurrent } \\
\text { CCT }\end{array}$ & 11 & 1 & 3.03 & .082 & 41.2 & 0.39 & -0.59 \\
\hline
\end{tabular}

Table 4: A summary of the calculated one-moderator models and associated significance levels. $Q_{0}$ is the Waldtype chi squared value for the omnibus test of model coefficients. $R^{2}$ is the pseudo $R^{2} . b_{0}$ and $b_{1}$ are the slope and intercept respectively. (ISI=inter-session interval in days, $A D M=$ Antidepressant Medication) 


\section{CURRENT META-ANALYSIS: Categorical treatment effects (Response and Remission rates)}

Data for response rates were available from 9 of the 11 effects (Figure 3a). The pooled LOR for response was positive, but did not reach significance $(k=9$, LOR $=0.36,95 \% \mathrm{CI}[-0.16,0.88], p=.176)$, Heterogeneity between studies did not exceed that expected by chance $(Q(8)=6.18, p=.627)$ and the $l^{2}$ statistic indicated that only $0.86 \%$ of the heterogeneity could not be explained by sampling error. Cumulative meta-analysis revealed that the meta-analytic combination of effects yielded an increase of precision from an MOE of 1.71 to 0.52 . Data for remission rates were available from 9 of the 11 effects (Figure 3b). Consistent with response rates, the pooled LOR for remission was positive, but did not reach significance ( $k=9$, LOR $=0.25,95 \% \mathrm{Cl}[-0.42,0.91], \mathrm{p}=.468$ ), Heterogeneity between studies did not exceed that expected by chance $(Q(8)=4.68, p=791)$ and the $l^{2}$ statistic indicated that all heterogeneity could be attributed to sampling error. Cumulative meta-analysis revealed that the meta-analytic combination of effects yielded an increase of precision from an MOE of 2.97 to 0.66 .

\subsection{Fail safe $\mathrm{N}$ analyses}

Owing to the non-significant summary effects for response and remission, we did not compute fail-safe $\mathrm{N}$ analyses based on a nominal significance level. Instead, we used the Orwin method to assess the impact of publication bias on effect size. These data are plotted in Figure 3c. 

(2)

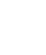

(n)

(n)

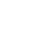
4 (2)

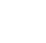
(n)

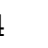
. , . . . . ,

\subsection{Publication bias}

Analyses revealed that no studies were trimmed and filled on the opposite side of zero in either the response or remission meta-analyses. Furthermore, both funnel plots were broadly symmetrical, suggesting a low risk of publication bias (figure $3 d \& 3 e$ ).

\subsection{Moderator analysis for categorical outcomes}

Meta regression revealed no statistically significant moderators of either response or remission rates.

\section{CURRENT META-ANALYSIS: SAFETY AND TOLERABILITY:}

Dropout rates were available from 9 studies (table 5) and were analysed in a random effects model using the log odds ratio as an effect size measure (effect sizes greater than 0 indicate a greater likelihood of dropout in the active relative to the sham tDCS group). The analysis revealed no significant differences in drop out rates ( $k=9$ $254 L O R=0.05,95 \% \mathrm{Cl}=[-1.0,1.10], p=.928)$. 
1

2

3

4

5

\begin{tabular}{lllll}
\hline Study & $N-$ & drop out rate due to & $N-$ & drop out rate due to
\end{tabular}$\quad p$

\begin{tabular}{|c|c|c|c|c|c|}
\hline Fregni $2006^{29}$ & 9 & 0 & 9 & 0 & 1 \\
\hline Boggio $2008^{26}$ & 21 & 0 & 19 & 0 & 1 \\
\hline Loo $2010^{30}$ & 20 & 0 & 20 & 0 & 1 \\
\hline Palm $2011^{33}$ & 11 & 0 & 11 & 0 & 1 \\
\hline Loo $2012^{34}$ & 33 & 1 & 31 & 1 & .964 \\
\hline Blumberger $2012^{36}$ & 13 & 1 & 11 & 0 & .558 \\
\hline Brunoni $2013^{27}$ & 60 & 3 & 60 & 1 & .347 \\
\hline Brunoni $2014^{42}$ & 20 & 7 & 17 & 3 & .245 \\
\hline Segrave $2014^{28}$ & 9 & 0 & 9 & 0 & 1 \\
\hline
\end{tabular}

Table 5 : Drop out rates due to adverse events in blind phase of tDCS depression RCTs 


\section{DISCUSSION}

We carried out a meta analysis of 10 RCTs comparing active tDCS to sham tDCS, including 393 participants with major depressive episodes in the context of unipolar or bipolar disorders. tDCS was used as mono therapy or as adjunctive treatment for depression in conjunction with medication and/or Cognitive Control Training (CCT). Analysis of continuous outcomes - depression rating scale scores, demonstrates clear superiority of active tDCS over sham tDCS in the treatment of MDE. The combined treatment effect was significant and consistent with a small effect size $(k=11, g=0.30,95 \% \mathrm{Cl}=[0.04,0.57], p=.027)$ (Figure 2$)$.

It is important to highlight the findings from our precision and power analyses: These indicate that for an individual study to detect the summary effect $(g=0.30)$ at the $p=.05$ level at $80 \%$ power, a total (combined treatment and control) $N$ of at least 346 would be required. The mean total sample size of the studies included in the meta analysis is 36 - it is therefore likely that the RCTs included lacked sufficient statistical power. Given that an $N$ of 346 is of considerable size, future studies may wish to focus on enhancing the precision of their interval based estimates within practical and financial constraints, rather than placing too much stock in conclusions based on null hypothesis significance testing. In this context, a priori power and precision analyses (and explicit reporting of the associated values) are recommended to enhance interpretation of data relating to tDCS efficacy in future RCTs. It is, of course, worth noting that power is not simply a function of sample size. Correspondingly, enhancements in the design of RCTs, including rigorous eligibility criteria and optimizing a sensitive study design and endpoint may decrease the number of participants required to detect an effect. The issues surrounding power, in turn, also go some way towards providing a possible explanation for the lack of statistically significant differences between active and sham tDCS in the categorical response and remission analyses that we carried out. Categorical outcome measures are more robust and clinically meaningful, but require a larger sample size to demonstrate an effect. We found that both response and remission rates showed a positive pooled LOR but failed to reach statistical significance at the $p=.05$ level. These findings are consistent with the findings from earlier meta-analyses: Kalu et al. (2012) ${ }^{12}$ used continuous outcome measures and found a significant superiority of active over sham tDCS; whereas Berlim et al. (2013) ${ }^{13}$, used categorical outcome measures and failed to demonstrate significant differences. The more recent meta analysis Shiozawa et al $(2014)^{14}$ used both types of outcome measures and demonstrated significant superiority of active over sham tDCS in both continuous and categorical outcome measures. This difference in findings is likely to be mainly due to the way we analyzed data from the largest tDCS trial to date ${ }^{27}$ - we decided to analyze the outcomes at week 2 , immediately following the 
50

daily tDCS treatment phase - in contrast to analyzing the outcomes at 6 weeks (the primary outcome measure for the trial), following 2 fortnightly "top-up" sessions. The rationale for our choice being that most other RCTs we included in the meta-analysis used an endpoint at the termination of the intensive tDCS treatment phase. The outcomes at week 6 demonstrate an added degree of separation between active and sham tDCS. Repeating our analyses with week 6 as an endpoint revealed that this is sufficient to provide overall statistical significance to the categorical analysis (response: LOR $=0.81,[0.28,1.34], p=.003$, remission: $\operatorname{LOR}=0.73,[0.131 .33], p=.017$ ) and slightly increase the magnitude of the continuous effect. $(g=0.37,[0.09,0.65], p=.008)$ This accords with the findings from other tDCS trials ${ }^{28,34,48}$, indicating that at follow up, participants' depression rating scales score continued to improve. We also decided to separately analyze the data from participants who received sertraline and those who received placebo medication in the Brunoni et al. 2013 trial $^{27}$ - this created two separate effects (hence although we included 10 RCTs, we analyzed $k=11$ effects). Moderator analysis demonstrated that although no moderators reached statistical significance at the level of $p=.05$, concurrent use of antidepressant medication, and concurrent use of Cognitive Control Training were the most important predictors, accounting for $47 \%$ and $36 \%$ of the total variation explained respectively (Figure 2e). Notably, these moderators remained the most important when outcomes for the Brunoni et al. 2013 trial $^{27}$ trial were analyzed at week 6, with the concurrent ADMs factor reaching significance $\left(R^{2}=24.15, Q(1)=4.94, p=.026\right)$.

Our moderator analyses revealed that samples who were not taking concurrent ADMs $(g=0.71,95 \% \mathrm{CI}[0.121 .29]$, $p=.019)$ had a larger treatment effect than those who were $(g=0.18,95 \% \mathrm{CI}[-0.160 .51], p=.302)$. Similarly, samples that did not receive concurrent CCT ( $g=0.39,95 \%$ CI[0.13 0.65], $p=.004)$ had a larger treatment effect than those who did ( $g=-0.20,95 \% \mathrm{CI}[-0.820 .41], p=.517)$. It is of note, that the largest trial to date ${ }^{27}$ included a group of participants, in whom tDCS and pharmacological antidepressant therapy were concurrently initiated - the authors report that this group did particularly well in terms of depression outcomes, and go on to postulate that simultaneous initiation of tDCS and ADM may confer added benefits in patients with MDE. One should note that these participants were treated with a low dose of antidepressant medication (sertraline $50 \mathrm{mg}$ daily). Insufficient data prevented us from conclusively analyzing the effect of treatment-resistance level as a moderator of tDCS outcomes. More trials are needed in order to improve the precision of moderator analyses; this is currently limited due to small $k$. The evidence suggests tDCS has a good safety and acceptability profile, with only mild adverse effects reported in most trials. There were no statistically significant differences in the drop-out rates between active and sham tDCS groups in any of the RCTs. The only serious adverse event recorded in published tDCS RCTs was a case of suicide, which was considered by the authors as unlikely to be directly related to tDCS ${ }^{30}$. Early reports of tDCS trials included descriptions of burns to the skin underlying scalp electrodes, but this has not been 
reported since researchers started using physiological saline rather than water to soak electrodes prior to use ${ }^{49}$. Several cases of tDCS-associated hypomanic episodes have been reported in the literature ${ }^{12,14,27}$.

This meta-analysis offers several improvements compared to previous publications (Table 2): The literature search extends up to April 2015, and therefore captures 3 new RCTs (43\% increase), which were not included in previous meta-analyses. This enabled us to increase the number of subjects by $23 \%$. We were able to assess the effect of adding tDCS to CCT; this was not previously meta-analytically addressed. The power to detect "small" effects is increased, as is the precision ( $21 \%$ improvement in pooled confidence interval width). We also performed power and precision analyses to directly inform future research in terms of sample size planning.

Limitations: The main limitation of this meta analysis is the low number of participants in most trials included. As demonstrated by our precision and power calculations, all but one of these trials ${ }^{27}$ are probably underpowered. This is likely to explain the lack of separation between active and sham tDCS in terms of categorical response and remission outcomes; as well as limiting the number of moderators reaching statistical significance. There is a lack of evidence regarding longer-term outcomes of tDCS in the acute and maintenance treatment of depression.

\section{Conclusions:}

Based on current evidence, the following conclusions may be drawn: First, tDCS may represent an effective treatment option for patients presenting with major depressive episodes. Second, tDCS offers a generally acceptable tolerability profile, which may make it a useful alternative to antidepressant medication in patients who do not wish to take medication and for those who cannot tolerate antidepressant medication. Third, the current body of evidence does not support the use of tDCS in treatment resistant depression. Fourth, the current body of evidence does not support the use of tDCS as an add-on augmentation treatment for depressed patient who are already taking an antidepressant or undergoing Cognitive Control Training (CCT). However, there may be an advantage for concurrently initiating treatment with an antidepressant and tDCS. Further research is needed, in particular, involving larger sample sizes over longer periods of treatment. 
a)
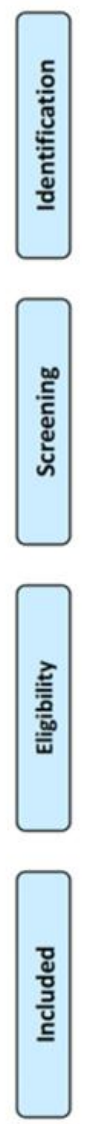

\# of additional records

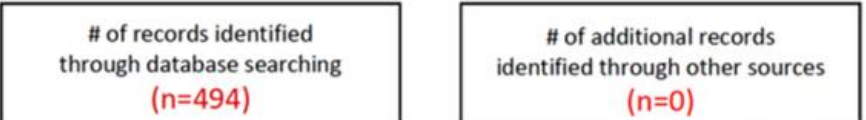

$(n=494)$

identified through other sources $(n=0)$

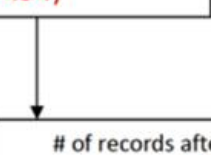

\# of records after duplicates removed $(n=494)$

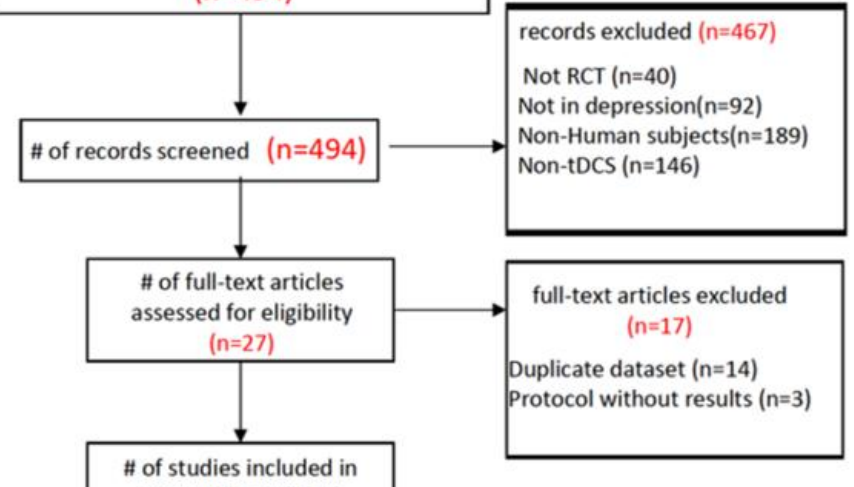

\# of studies included in

$$
\begin{array}{r}
\text { qualitative syn } \\
(\mathrm{n}=10)
\end{array}
$$

\section{\# of studies included in}

quantitative synthesis b)

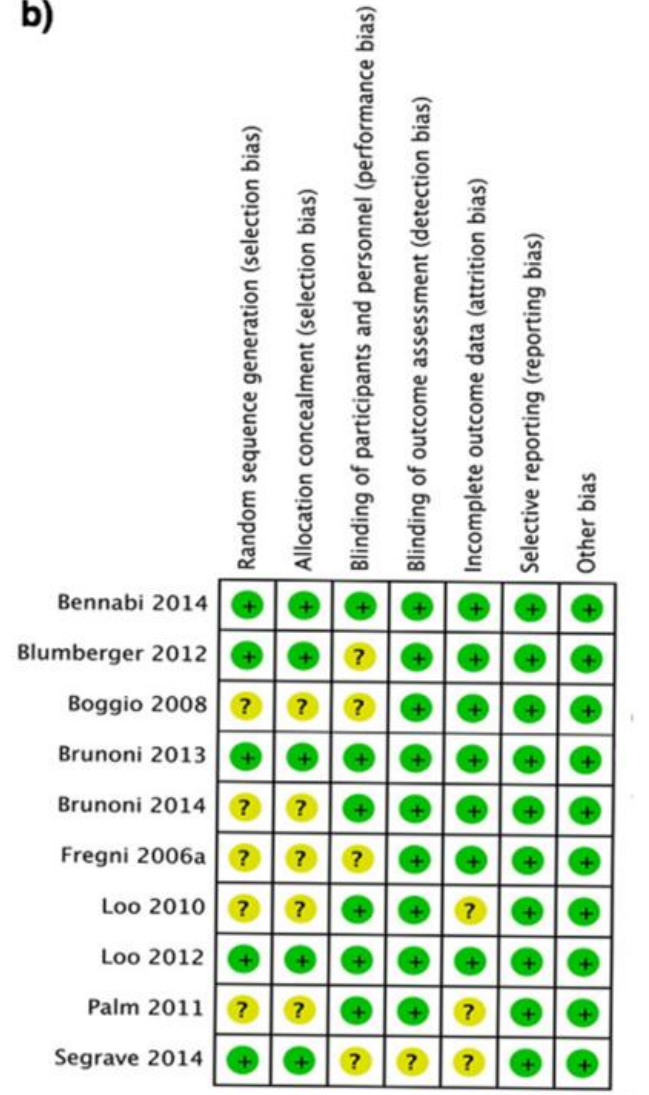

38348

40349

41350

43351

44351

45352

46

47353

48

Figure 1 Study selection and quality assurance: (a) PRISMA ${ }^{1}$ study-selection flowchart for our systematic review and meta-analysis. RCT = Randomised Controlled Trial. (b) Summary of risk of bias in line with the Cochrane Collaboration's tool for assessing risk of bias in randomised trials ${ }^{25}$. Green circles represent low risk; yellow circles represent unclear risk of bias. The summary was generated using Cochrane's Review Manager software (RevMan5.3) -http://tech.cochrane.org/revm 

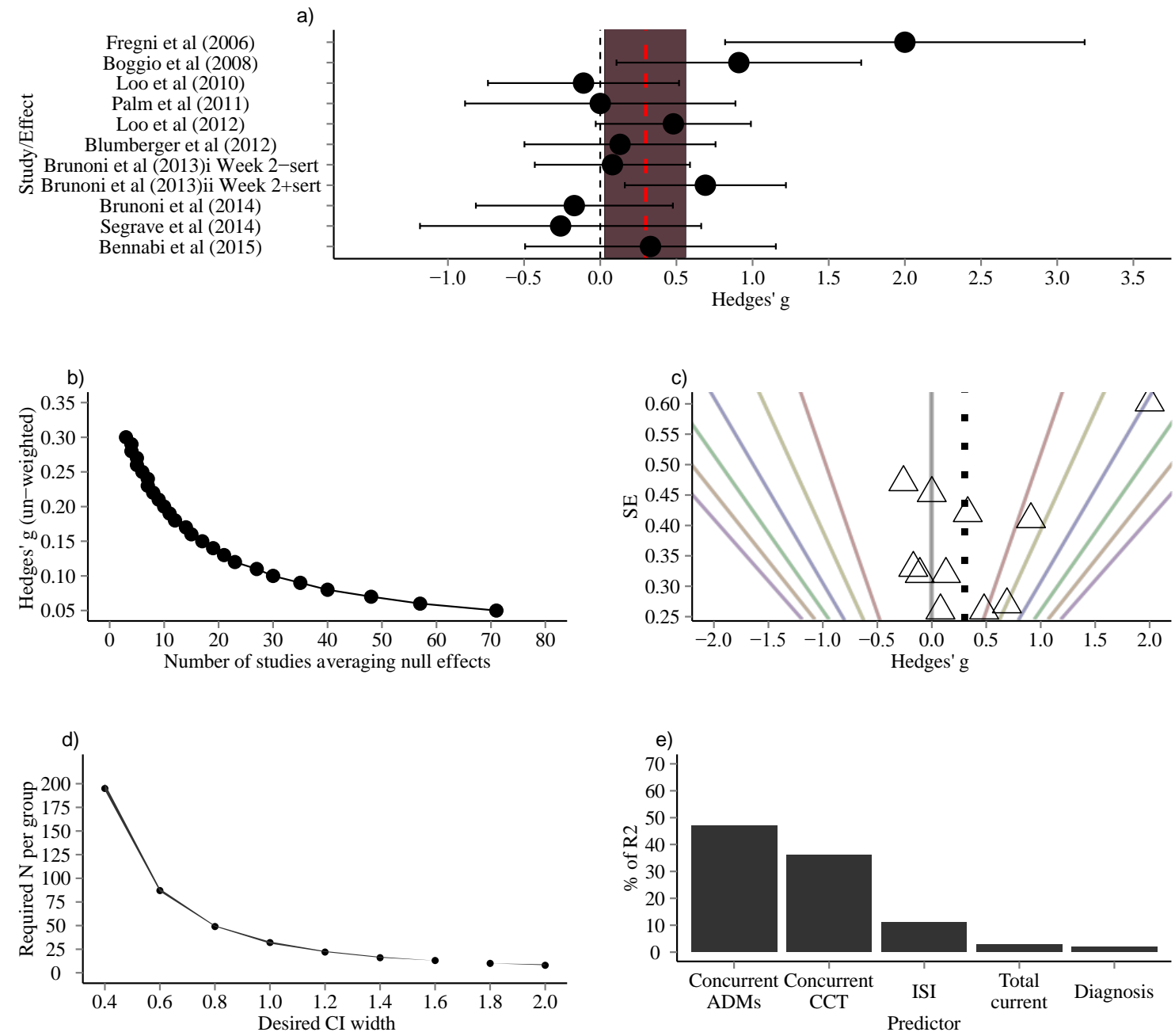

Figure 2. (a) Forest plot of effect sizes for active versus sham treatment. Error bars are $95 \%$ confidence intervals. Dotted red line depicts pooled effect. Shaded red region is $95 \%$ confidence interval. (b) Orwin fail-safe $\mathrm{N}$ analyses (c) Funnel plot. Dotted line is the pooled effect size, colored lines are $p$ values (e.g. red $=.05$, yellow $=.01$, blue $=$ .001). (d) Outcome of precision analyses. For instance, to have $99 \%$ assurance that a $95 \% \mathrm{Cl}$ will be less than 0.8 , approximately 50 participants per group would be required. (e) Relative importance of each moderator. Note that total TDCS time, amplitude, session length and number of sessions were removed due to multi-colinearity. Total current is instead included to summarize these variables. 
2

3

4

5

6

7

8

9

10

11

12

13

14

15

16

17

18

19

20

21

22

23

24

25

26

27

28

29

30

31

32

33

34

35

36

37

38

39

40

41

42

43

44

45

46

47

48362

49

50

51

52

53

54

55

56

57

58

59

60

61

62

63

64

65
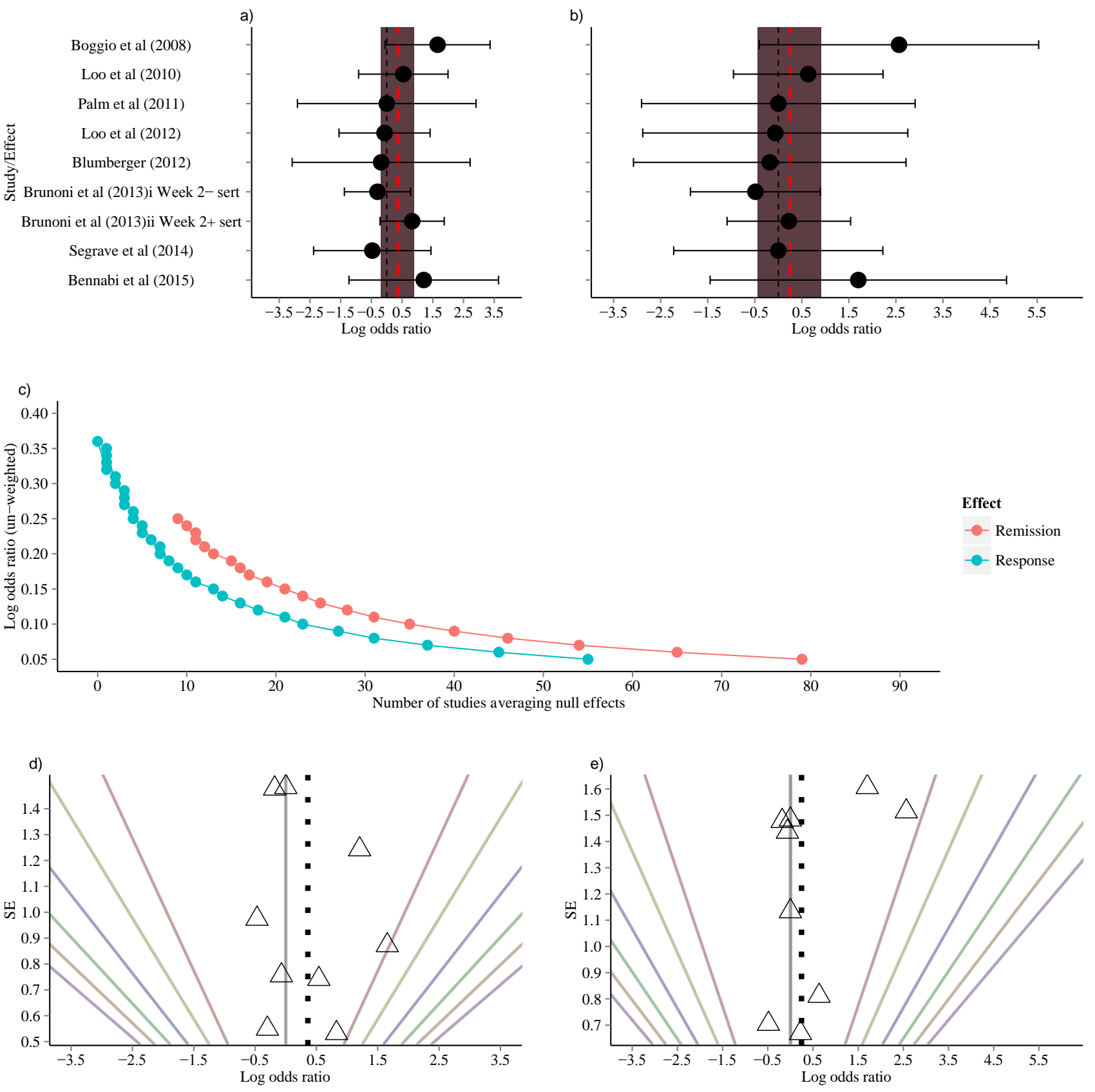

Page 39 of 52 
2

3

4363

5

6364

8365

9 10

11

12

13

14368

15369

16

17

18

19

20

21

22

23

24

25

26

27

28

29

30

31

32

33

34

35

36

37

38

39

40

41

42

43

44

45

46

47

48

49

50

51

52

53

54

55

56

57

58

59

60

61

62

63

64

65

Figure 3. (a) Forest plot of effect sizes for active versus sham treatment: response rates. (b) Forest plot of effect sizes for active versus sham treatment: remission rates. Error bars are $95 \%$ confidence intervals. Dotted red line depicts pooled effect. Shaded red region is $95 \%$ confidence interval. (c) Orwin fail-safe $\mathrm{N}$ analyses (d) Funnel plot: response rates. (d) Funnel plot: remission rates. Dotted line is the pooled effect size, colored lines are $p$ values (e.g. red $=.05$, yellow $=.01$, blue $=.001)$. 
7

Liberati A, Altman DG, Tetzlaff J, et al. The PRISMA statement for reporting systematic reviews and meta-analyses of studies that evaluate healthcare interventions: explanation and elaboration. BMJ. 2009;339(jul21 1):b2700-b2700. doi:10.1136/bmj.b2700.

2. Kessler RC, Berglund P, Demler O, et al. The Epidemiology of Major Depressive Disorder: Results From the National Comorbidity Survey Replication (NCS-R). JAMA. 2003;289(23):3095-3105. doi:10.1001/jama.289.23.3095.

3. Prince M, Patel V, Saxena S, et al. No health without mental health. The Lancet. 2007;370(9590):859877.

4. Rush A, Trivedi M, Wisniewski S, et al. Acute and longer-term outcomes in depressed outpatients requiring one or several treatment steps: a STAR* D report. American Journal of Psychiatry. 2006;163(11): 1905-1917.

5. Anderson I, Ferrier I, Baldwin R, et al. Evidence-based guidelines for treating depressive disorders with antidepressants: A revision of the 2000 British Association for Psychopharmacology guidelines. Journal of Psychopharmacology. 2008;22(4):343-396. doi:10.1177/0269881107088441.

6. NICE. Depression: the Treatment and Management of Depression in Adults (Update).

. niceorguk. 2009. http://www.nice.org.uk/guidance/cg90/evidence/cg90-depression-in-adults-full-guidance2. Accessed June 4, 2015.

Priori A, Hallett M, Rothwell JC. Repetitive transcranial magnetic stimulation or transcranial direct current stimulation? Brain Stimul. 2009;2(4):241-245. doi:10.1016/j.brs.2009.02.004.

8. Ruohonen J, Karhu J. tDCS possibly stimulates glial cells. Clinical neurophysiology. 2012;123(10):2006-2009. doi:10.1016/j.clinph.2012.02.082.

9. Brunoni AR, Nitsche MA, Bolognini N, et al. Clinical research with transcranial direct current stimulation (tDCS): Challenges and future directions. Brain Stimul. 2012;5(3):175-195. doi:10.1016/j.brs.2011.03.002.

10. Nitsche MA, Boggio PS, Fregni F, Pascual-Leone A. Treatment of depression with transcranial direct current stimulation (tDCS): A Review. Experimental Neurology. 2009;219(1):14-19. doi:10.1016/j.expneurol.2009.03.038.

11. Brunoni AR, Ferrucci R, Fregni F, Boggio PS, Priori A. Transcranial direct current stimulation for the treatment of major depressive disorder: A summary of preclinical, clinical and translational findings. Progress in Neuropsychopharmacology \& Biological Psychiatry. 2012;39(1):9-16. doi:10.1016/j.pnpbp.2012.05.016.

12. Kalu UG, Sexton CE, Loo CK, Ebmeier KP. Transcranial direct current stimulation in the treatment of major depression: a meta-analysis. Psychol Med. 2012:1-10. doi:10.1017/S0033291711003059.

13.

Berlim MT, Van den Eynde F, Daskalakis ZJ. Clinical utility of transcranial direct current stimulation (tDCS) for treating major depression: A systematic review and meta-analysis of randomized, doubleblind and sham-controlled trials. Journal of Psychiatric Research. 2013;47(1):1-7. doi:10.1016/j.jpsychires.2012.09.025.

14. Shiozawa P, Fregni F, Benseñor IM, et al. Transcranial direct current stimulation for major depression: an updated systematic review and meta-analysis. The International Journal of Neuropsychopharmacology. 2014;17(9):1443-1452. doi:10.1017/S1461145714000418. 
Chandler J, Churchill R, Higgins J. Methodological standards for the conduct of new Cochrane Intervention Reviews Version 2.1. The Cochrane Library. 2011.

741416.

20.

21.

23.

26.

Cumming G. Understanding the New Statistics. Routledge; 2012.

Schmidt FL, Oh I-S, Hayes TL. Fixed-versus random-effects models in meta-analysis: model properties and an empirical comparison of differences in results. Br J Math Stat Psychol. 2009;62(Pt 1):97-128. doi:10.1348/000711007X255327.

López-López JA, Marín-Martínez F, Sánchez-Meca J, Van den Noortgate W, Viechtbauer W. Estimation of the predictive power of the model in mixed-effects meta-regression: A simulation study. $\mathrm{Br} J$ Math Stat Psychol. 2013;67(1):30-48. doi:10.1111/bmsp.12002.

19. Raudenbush SW. Random effects models. The handbook of research synthesis. 1994. doi:10.1002/9780470755839.ch5.

Viechtbauer W. Conducting meta-analyses in R with the metafor package. Journal of Statistical Software. 2010.

Orwin RG. A fail-safe $\mathrm{N}$ for effect size in meta-analysis. Journal of educational statistics. 1983;8(2):157. doi:10.2307/1164923.

Rosenthal R. Meta-Analytic Procedures for Social Research. SAGE; 1991.

Kelley K, Rausch JR. Sample size planning for the standardized mean difference: accuracy in parameter estimation via narrow confidence intervals. Psychol Methods. 2006;11(4):363-385. doi:10.1037/1082-989X.11.4.363.

Kelley K. Confidence intervals for standardized effect sizes: Theory, application, and implementation. Journal of Statistical Software. 2007.

Higgins JPT, Altman DG, Gøtzsche PC, et al. The Cochrane Collaboration's tool for assessing risk of bias in randomised trials. BMJ. 2011;343:d5928-d5928. doi:10.1136/bmj.d5928.

Boggio PS, Rigonatti SP, Ribeiro RB, et al. A randomized, double-blind clinical trial on the efficacy of cortical direct current stimulation for the treatment of major depression. Int J Neuropsychopharmacol. 2008;11(02):249-254. doi:10.1017/S1461145707007833.

Brunoni AR. The Sertraline vs Electrical Current Therapy for Treating Depression Clinical Study. JAMA Psychiatry. 2013;70(4):383-391. doi:10.1001/2013.jamapsychiatry.32.

Segrave RA, Arnold S, Hoy K, Fitzgerald PB. Concurrent cognitive control training augments the antidepressant efficacy of tDCS: a pilot study. Brain Stimul. 2014;7(2):325-331. doi:10.1016/j.brs.2013.12.008.

Fregni F, Boggio PS, Nitsche MA, Rigonatti SP, Pascual-Leone A. Cognitive effects of repeated sessions of transcranial direct current stimulation in patients with depression. Depress Anxiety. 2006;23(8):482-484. doi:10.1002/da.20201.

Loo CK, Sachdev P, Martin D, et al. A double-blind, sham-controlled trial of transcranial direct current stimulation for the treatment of depression. The International Journal of Neuropsychopharmacology. 2010;13(1):61-69. doi:10.1017/S1461145709990411.

Fregni F, Boggio PS, Nitsche MA, Marcolin MA, Rigonatti SP, Pascual-Leone A. Treatment of major depression with transcranial direct current stimulation. Bipolar Disorders. 2006;8(2):203-204. doi:10.1111/j.1399-5618.2006.00291.x. 
Palm U, Schiller C, Fintescu Z, et al. Transcranial direct current stimulation in treatment resistant depression: a randomized double-blind, placebo-controlled study. Brain Stimul. 2011:1-10. doi:10.1016/j.brs.2011.08.005.

Loo CK, Alonzo A, Martin D, Mitchell PB, Galvez V, Sachdev P. Transcranial direct current stimulation for depression: 3-week, randomised, sham-controlled trial. The British Journal of Psychiatry. 2012;200(1):52-59. doi:10.1192/bjp.bp.111.097634.

35. Slotema CW, Blom JD, Hoek HW, Sommer IEC. Should we expand the toolbox of psychiatric treatment methods to include Repetitive Transcranial Magnetic Stimulation (rTMS)? A meta-analysis of the efficacy of rTMS in psychiatric disorders. The Journal of clinical psychiatry. 2010;71(7):873-884. doi:10.4088/JCP.08m04872gre.

Blumberger DM, Tran LC, Fitzgerald PB, Hoy KE, Daskalakis ZJ. A randomized double-blind shamcontrolled study of transcranial direct current stimulation for treatment-resistant major depression. Front Psychiatry. 2012;3:74. doi:10.3389/fpsyt.2012.00074.

Bennabi D, Nicolier M, Monnin J, et al. Pilot study of feasibility of the effect of treatment with tDCS in patients suffering from treatment-resistant depression treated with escitalopram. Clinical neurophysiology : official journal of the International Federation of Clinical Neurophysiology. 2015;126(6):1185-1189. doi:10.1016/j.clinph.2014.09.026.

38.

39.

40.

41.

42.

43.

44.

45.

46.

47.

Andrews SC, Hoy KE, Enticott PG, Daskalakis ZJ, Fitzgerald PB. Improving working memory: the effect of combining cognitive activity and anodal transcranial direct current stimulation to the left dorsolateral prefrontal cortex. Brain Stimul. 2011;4(2):84-89. doi:10.1016/j.brs.2010.06.004.

Kadosh RC, Soskic S, luculano T, Kanai R, Walsh V. Modulating Neuronal Activity Produces Specific and Long-Lasting Changes in Numerical Competence. Current Biology. 2010;20(22):2016-2020. doi:10.1016/j.cub.2010.10.007.

Vercammen A, Rushby JA, Loo C, Short B, Weickert CS, Weickert TW. Transcranial direct current stimulation influences probabilistic association learning in schizophrenia. Schizophrenia Research. 2011;131(1-3):198-205. doi:10.1016/j.schres.2011.06.021.

Martin DM, Liu R, Alonzo A, et al. Can transcranial direct current stimulation enhance outcomes from cognitive training? A randomized controlled trial in healthy participants. Int J Neuropsychopharmacol. 2013;16(9):1927-1936. doi:10.1017/S1461145713000539.

Brunoni AR, Boggio PS, De Raedt R, et al. Cognitive control therapy and transcranial direct current stimulation for depression: a randomized, double-blinded, controlled trial. Journal of Affective Disorders. 2014;162:43-49. doi:10.1016/j.jad.2014.03.026.

Martin DM, Alonzo A, Mitchell PB, Sachdev P, Gálvez V, Loo CK. Fronto-extracephalic transcranial direct current stimulation as a treatment for major depression: an open-label pilot study. Journal of Affective Disorders. 2011;134(1-3):459-463. doi:10.1016/j.jad.2011.05.018.

Brunoni AR, Valiengo L, Baccaro A, et al. Sertraline vs. ELectrical Current Therapy for Treating Depression Clinical Trial--SELECT TDCS: design, rationale and objectives. Contemp Clin Trials. 2011;32(1):90-98. doi:10.1016/j.cct.2010.09.007.

Fava M, Rush AJ, Trivedi MH, et al. Background and rationale for the sequenced treatment alternatives to relieve depression (STAR*D) study. PSC. 2003;26(2):457-94-x.

Palm U, Keeser D, Schiller C, et al. Skin lesions after treatment with transcranial direct current stimulation (tDCS). Brain Stimul. 2008;1(4):386-387. doi:10.1016/j.brs.2008.04.003.

Duval S, Duval S, Tweedie R, Tweedie R. A nonparametric "trim and fill" method of accounting for publication bias in meta-analysis. Journal of the American Statistical .... 2000;95(449):89-98. doi:10.1080/01621459.2000.10473905. 
1

2

3

4499

5500

6501

7

8502

9503

10504

11505

12

13506

14

15507

16

17

18

19

20

21

22511

23

24512

25

26513

27

28

29

30

31

50

51

52

53

54

55

56

57

58

59

60

61

62

63

516

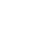

48. Brunoni AR, Ferrucci R, Bortolomasi M, et al. Transcranial direct current stimulation (tDCS) in unipolar vs. bipolar depressive disorder. Progress in Neuro-Psychopharmacology and Biological Psychiatry. 2011;35(1):96-101. doi:10.1016/j.pnpbp.2010.09.010.

49. Brunoni AR, Amadera J, Berbel B, Volz MS, Rizzerio BG, Fregni F. A systematic review on reporting and assessment of adverse effects associated with transcranial direct current stimulation. The International Journal of Neuropsychopharmacology. 2011;14(8):1133-1145. doi:10.1017/S1461145710001690.

Conflict of Interest disclosures:

- None declared

Funding sources:

None declared 


\begin{tabular}{|c|c|c|}
\hline Potential moderator & Units & Descriptive Statistics \\
\hline Current & mA (Boolean) & $\begin{array}{l}k=11 \\
1 \mathrm{~mA}(k=2) \\
2 \mathrm{~mA}(k=9)\end{array}$ \\
\hline Course delivered & Number of sessions & $\begin{array}{l}k=11 \\
M=9.55 \\
S D=3.50 \\
\text { Range }=5-15\end{array}$ \\
\hline Session duration & Minutes per session (Boolean) & $\begin{array}{l}k=11 \\
20 \operatorname{mins}(k=7) \\
30 \operatorname{mins}(k=4)\end{array}$ \\
\hline Total tDCS time & $\begin{array}{l}\text { Number of sessions } x \text { session } \\
\text { duration (minutes) }\end{array}$ & $\begin{array}{l}k=11 \\
M=229.09 \\
S D=87.80 \\
\text { Range }=100-300\end{array}$ \\
\hline Total current delivered & $\begin{array}{l}\mathrm{mA} \times \text { number of sessions } \mathrm{x} \text { session } \\
\text { duration }\end{array}$ & $\begin{array}{l}k=11 \\
M=430.91 \\
S D=210.97 \\
\text { Range }=100-600\end{array}$ \\
\hline Inter-session intervals & Days (Boolean) & $\begin{array}{l}k=11 \\
1 \text { day }(k=9) \\
2 \text { days }(k=2)\end{array}$ \\
\hline Diagnosis & & $\begin{array}{l}k=11 \\
\text { unipolar }(k=9) \\
\text { unipolar and bipolar }(k=2)\end{array}$ \\
\hline $\begin{array}{l}\text { Concurrent anti-depressant } \\
\text { medications (ADMs) }\end{array}$ & & $\begin{array}{l}k=11 \\
\text { Concurrent ADMs }(k=8) \\
\text { No-concurrent } \operatorname{ADMs}(k=3\end{array}$ \\
\hline $\begin{array}{l}\text { Concurrent cognitive control } \\
\text { therapy }(\mathrm{CCT})\end{array}$ & & $\begin{array}{l}k=11 \\
\text { Concurrent CCT }(k=2) \\
\text { No concurrent CCT }(k=9)\end{array}$ \\
\hline Treatment resistance level & $\begin{array}{l}\text { Number of previous courses of } \\
\text { antidepressants failed in current } \\
\text { episode }\end{array}$ & $k=9($ see table 3$)$ \\
\hline
\end{tabular}

2 Table 1: variables examined as potential moderators of tDCS outcomes in depression. These include both

3 participant-related and tDCS-related factors, with a view to enable meta-analytical examination of the variables

4 found to be associated with tDCS outcomes in the narrative analysis. 


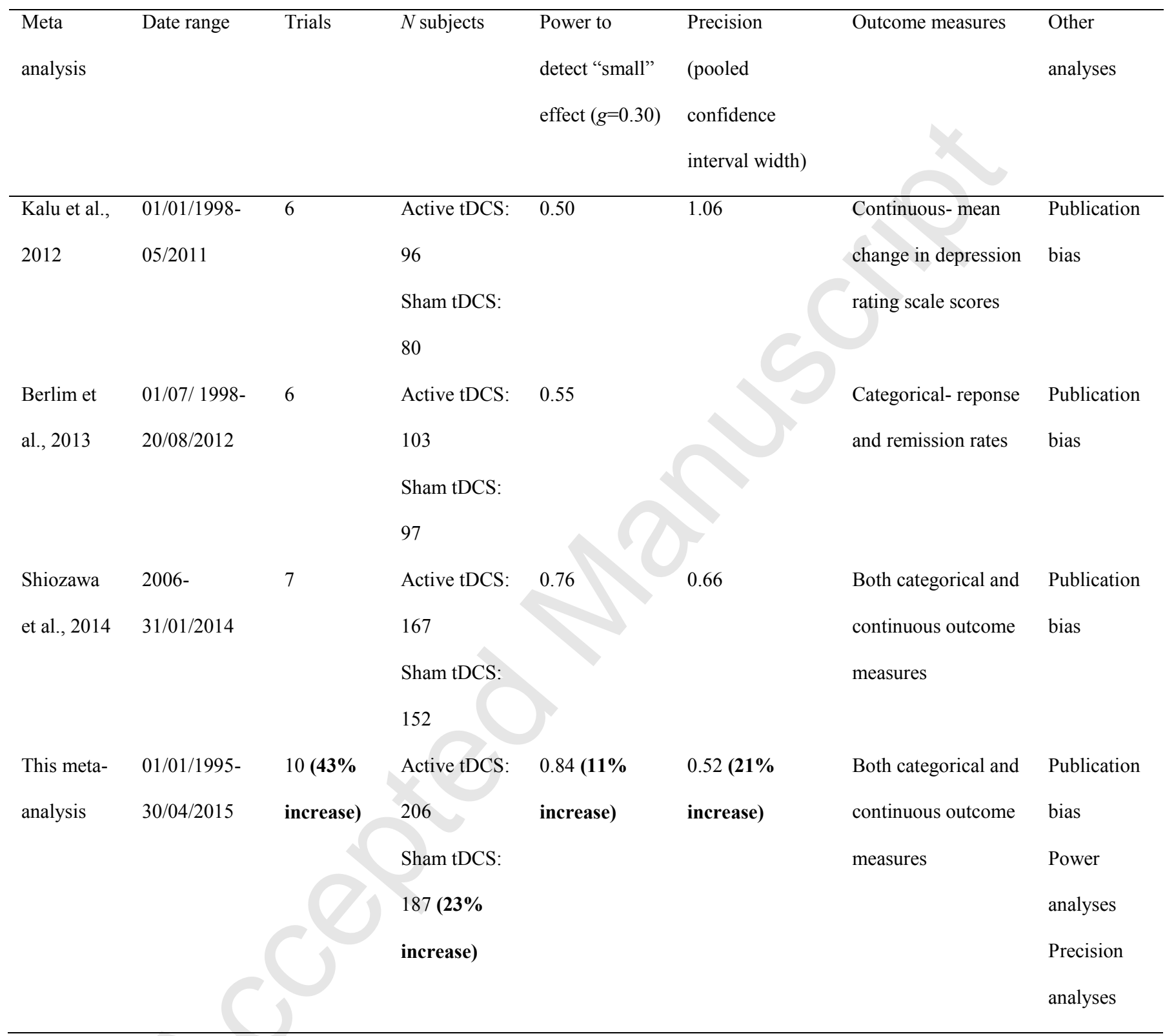

7

8 Table 2: Comparison of previously published and current meta-analyses of tDCS in depression 


\begin{tabular}{|c|c|c|c|c|c|c|c|c|c|c|c|c|c|}
\hline Name & $\begin{array}{l}\text { Diagnosi } \\
\mathrm{s}\end{array}$ & $\begin{array}{l}\mathrm{N} \\
\text { active, } \\
\text { sham }\end{array}$ & $\begin{array}{l}\text { Age } \\
\text { (S.D.) }\end{array}$ & $\begin{array}{l}\text { Gender } \\
\% \\
\text { Female }\end{array}$ & $\begin{array}{l}\text { Rating } \\
\text { Scale }\end{array}$ & $\begin{array}{l}\text { tDCS } \\
\text { Curren } \\
t(m A)\end{array}$ & $\begin{array}{l}\text { tDCS } \\
\text { Session } \\
\text { Duration } \\
\text { (min) }\end{array}$ & $\begin{array}{l}\text { tDCS } \\
\text { Montage: } \\
\text { anode, } \\
\text { cathoode }\end{array}$ & $\begin{array}{l}\text { Number of } \\
\text { tDCS } \\
\text { Sessions }\end{array}$ & $\begin{array}{l}\text { Treatment } \\
\text { resistance } \\
\text { level }\end{array}$ & $\begin{array}{l}\text { Con- } \\
\text { current } \\
\text { ADM }\end{array}$ & $\begin{array}{l}\text { Con- } \\
\text { current } \\
\text { CCT }\end{array}$ & $\begin{array}{l}\text { Depression } \\
\text { outcome }\end{array}$ \\
\hline Fregni $2006^{29}$ & Unipolar & $\overline{9,9}$ & $48.2(10)$ & No data & MADRS & 1.0 & 20 & F3, FP2 & 5 & no data & No & No & Active $>$ Sham \\
\hline Boggio $2008^{26}$ & Unipolar & 21,10 & $49(7.4)$ & $67.5 \%$ & HDRS & 2.0 & 20 & F3, FP2 & 10 & 1.7 & No & No & Active $>$ Sham \\
\hline Loo $2010^{30}$ & Unipolar & 20,20 & $\begin{array}{c}47.3 \\
(11.3)\end{array}$ & $55 \%$ & MADRS & 1.0 & 20 & F3, FP2 & 5 & $\begin{array}{l}\text { Active } 1.0 \\
\text { Sham } 1.7\end{array}$ & Yes & No & Active=Sham \\
\hline Palm 2011 33 & $\begin{array}{l}\text { unipolar } \\
\& \text { bipolar }\end{array}$ & 11,11 & $57(12)$ & $50 \%$ & HDRS & $\begin{array}{l}1.0 \text { or } \\
2.0\end{array}$ & 20 & F3, FP2 & 10 & $\begin{array}{l}\text { Active } 2.9 \\
\text { Sham } 2.91\end{array}$ & Yes & No & Active $=$ Sham \\
\hline Loo $2012^{34}$ & $\begin{array}{l}\text { unipolar } \\
\text { \& bipolar }\end{array}$ & 33,31 & $\begin{array}{c}48.2 \\
(12.5)\end{array}$ & $46.6 \%$ & MADRS & 2.0 & 20 & F3, F8 & 15 & $\begin{array}{l}\text { Active } 1.71 \\
\text { Sham } 1.79\end{array}$ & Yes & No & Active $>$ Sham \\
\hline $\begin{array}{l}\text { Blumberger } \\
2012^{36}\end{array}$ & Unipolar & 13,11 & $\begin{array}{c}42.7 \\
(11.6)\end{array}$ & $45.6 \%$ & HDRS & 2.0 & 20 & F3, F4 & 15 & $\begin{array}{l}\text { Active } 4.3 \\
\text { Sham } 4.1\end{array}$ & Yes & No & Active $=$ Sham \\
\hline Brunoni $2013^{27}$ & Unipolar & 30,30 & $42(12)$ & $68 \%$ & MADRS & 2.0 & 30 & $\mathrm{~F} 3, \mathrm{~F} 4$ & $10+2$ & 1.7 & Yes & No & Active $>$ Sham \\
\hline Bennabi2014 ${ }^{37}$ & Unipolar & 12,12 & $\begin{array}{c}61.8 \\
(16.3)\end{array}$ & $75 \%$ & HDRS & 2.0 & 30 & F3, FP2 & $\begin{array}{l}10 \text { ( } 5 \text { days } \\
\text { twice daily) }\end{array}$ & $100 \%>2$ & Yes & No & Active $=$ Sham \\
\hline Segrave $2014^{28}$ & Unipolar & 9,9 & $\begin{array}{c}40.4 \\
(14.5)\end{array}$ & $37 \%$ & MADRS & 2.0 & 24 & $\mathrm{~F} 3, \mathrm{~F} 8$ & 5 & 3.0 & Yes & Yes & Active $=$ Sham \\
\hline Brunoni $2014^{42}$ & Unipolar & 20,17 & $18-65$ & $70.2 \%$ & HDRS & 2.0 & 30 & F3, F4 & 10 & $35-41 \%>2$ & Yes & Yes & Active $=$ Sham \\
\hline
\end{tabular}

10 Table 3: Participant-related factors, tDCS-related factors and tDCS trial depression outcomes: RCTs of tDCS in depression listed in chronological

11 order. Depression outcome is a categorical statement as to whether active tDCS out-preformed sham tDCS in terms of depression outcome

12 measures $(p \leq 0.05)$, at the end of active treatment course. ADM = Antidepressant Medication. CCT=Cognitive Control Training. The degree of

13 treatment resistance is indicated by the number of ADM trials failed prior to starting tDCS. 


\begin{tabular}{|c|c|c|c|c|c|c|c|}
\hline Moderator & $k$ & $d f$ & Qo & $p$ & $R^{2}$ & $b_{0}$ & $b_{1}$ \\
\hline Current & 11 & 1 & 0.50 & .481 & 0.0 & 0.57 & -0.30 \\
\hline $\begin{array}{l}\text { Number of } \\
\text { sessions }\end{array}$ & 11 & 1 & 0.00 & .964 & 0.0 & 0.28 & 0.00 \\
\hline $\begin{array}{l}\text { Session } \\
\text { length }\end{array}$ & 11 & 1 & 014 & .705 & 0.0 & 0.35 & -0.11 \\
\hline $\begin{array}{l}\text { Total tDCS } \\
\text { time }\end{array}$ & 11 & 1 & 0.07 & .791 & 0.0 & 0.43 & -0.00 \\
\hline Total current & 11 & 1 & 0.41 & .783 & 0.0 & 0.41 & -0.00 \\
\hline ISI & 11 & 1 & $0.50-$ & .481 & 0.0 & 0.26 & 0.30 \\
\hline Diagnosis & 11 & 1 & 0.00 & .995 & 0.0 & 0.31 & -0.00 \\
\hline $\begin{array}{l}\text { Concurrent } \\
\text { ADMs }\end{array}$ & 11 & 1 & 2.35 & .125 & 0.0 & 0.70 & -0.53 \\
\hline $\begin{array}{l}\text { Concurrent } \\
\text { CCT }\end{array}$ & 11 & & 3.03 & .082 & 41.2 & 0.39 & -0.59 \\
\hline
\end{tabular}

Table 4: A summary of the calculated one-moderator models and associated significance levels. $Q_{0}$ is the Wald-type chi squared value for the omnibus test of model coefficients. $R^{2}$ is the pseudo $R^{2} . b_{0}$ and $b_{1}$ are the slope and intercept respectively. (ISI=inter-session interval in days, ADM = Antidepressant Medication) 


\begin{tabular}{|c|c|c|c|c|}
\hline Study & $\begin{array}{l}n- \\
\text { active }\end{array}$ & $\begin{array}{l}\text { drop out rate due to } \\
\text { adverse effects - } \\
\text { active tDCS }\end{array}$ & $\begin{array}{l}\mathrm{n}- \\
\text { control }\end{array}$ & $\begin{array}{l}\text { drop out rate due to } \\
\text { adverse effects - sham } \\
\text { tDCS }\end{array}$ \\
\hline
\end{tabular}

\begin{tabular}{|c|c|c|c|c|c|}
\hline Fregni $2006^{29}$ & 9 & 0 & 9 & 0 & 1 \\
\hline Boggio $2008^{26}$ & 21 & 0 & 19 & 0 & 1 \\
\hline Loo $2010^{30}$ & 20 & 0 & 20 & 0 & 1 \\
\hline Palm $2011^{33}$ & 11 & 0 & 11 & 0 & 1 \\
\hline Loo $2012^{34}$ & 33 & 1 & 31 & 1 & .964 \\
\hline Blumberger $2012^{36}$ & 13 & 1 & 11 & 0 & .558 \\
\hline Brunoni $2013^{27}$ & 60 & 3 & 60 & 1 & .347 \\
\hline Brunoni $2014^{42}$ & 20 & 1 & 17 & 3 & .245 \\
\hline Segrave $2014^{28}$ & 9 & 0 & 9 & 0 & 1 \\
\hline
\end{tabular}

Table 5 : Drop out rates due to adverse events in blind phase of tDCS depression RCTs 
a)

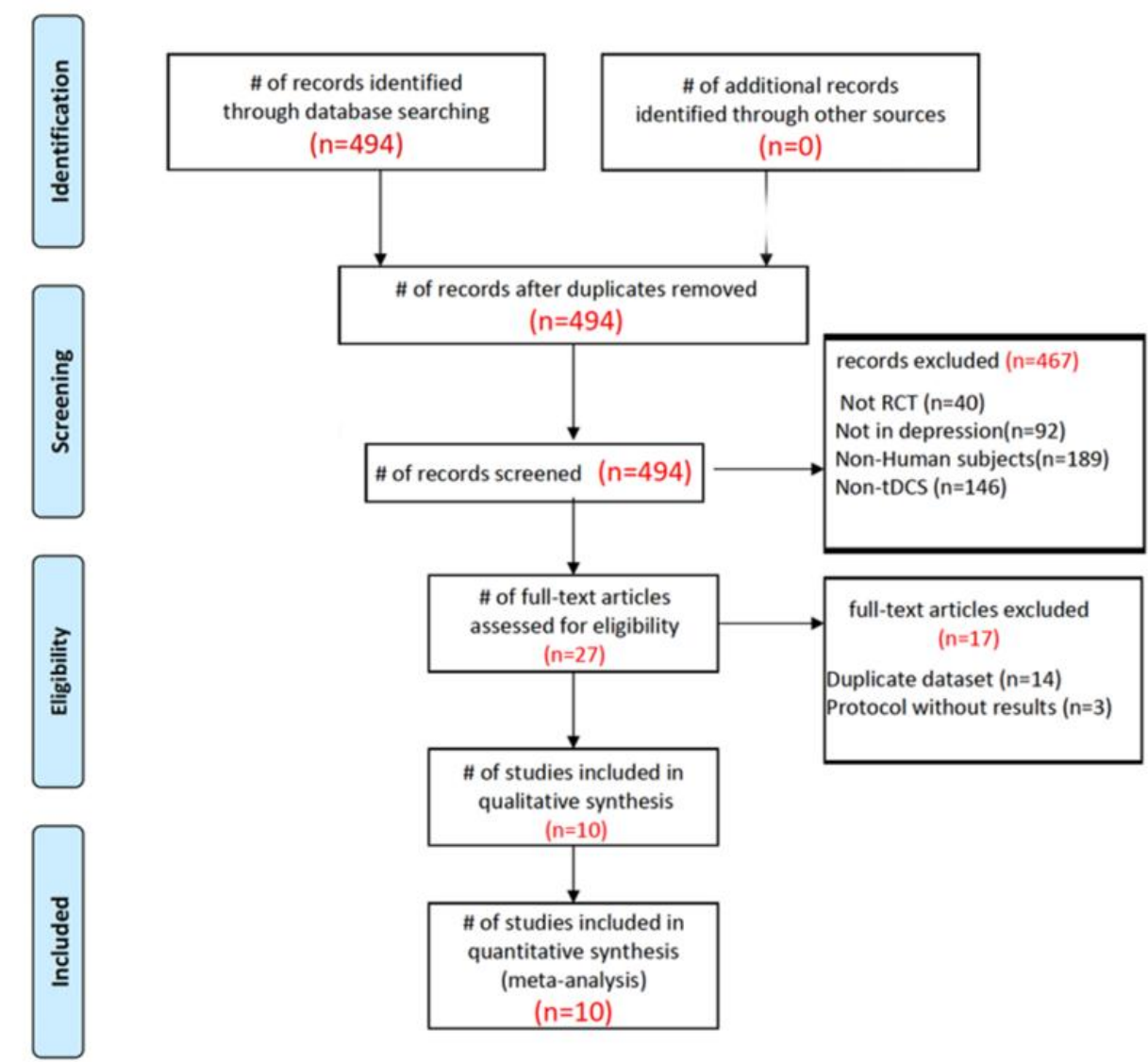

b)

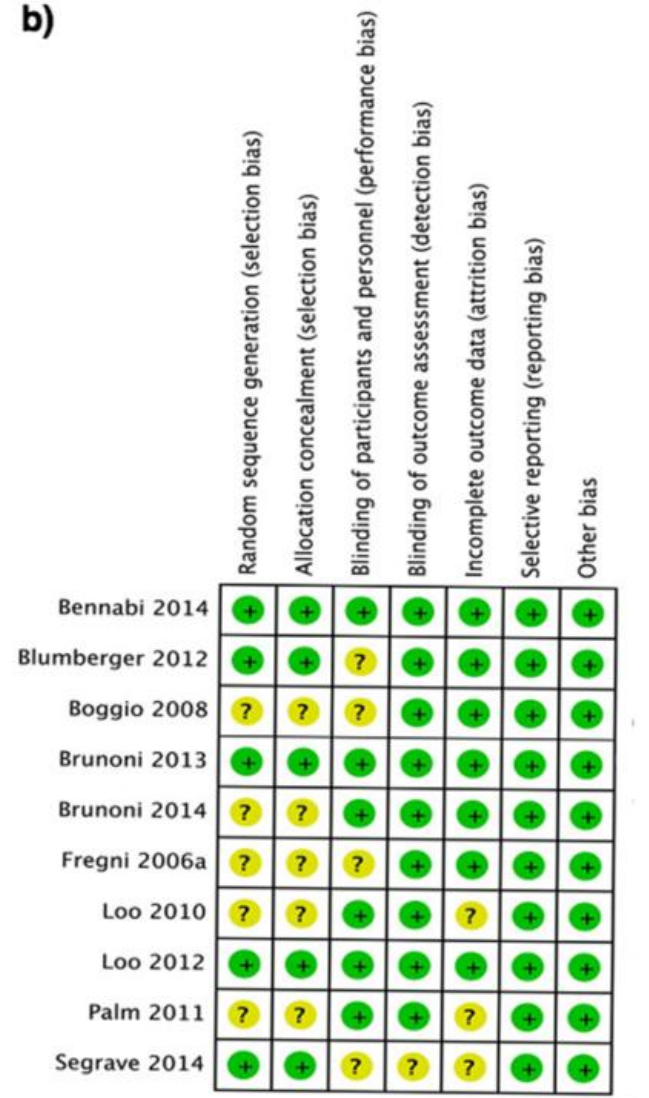

Figure 1 Study selection and quality assurance: (a) PRISMA ${ }^{1}$ study-selection flowchart for our systematic review and meta-analysis. RCT = 

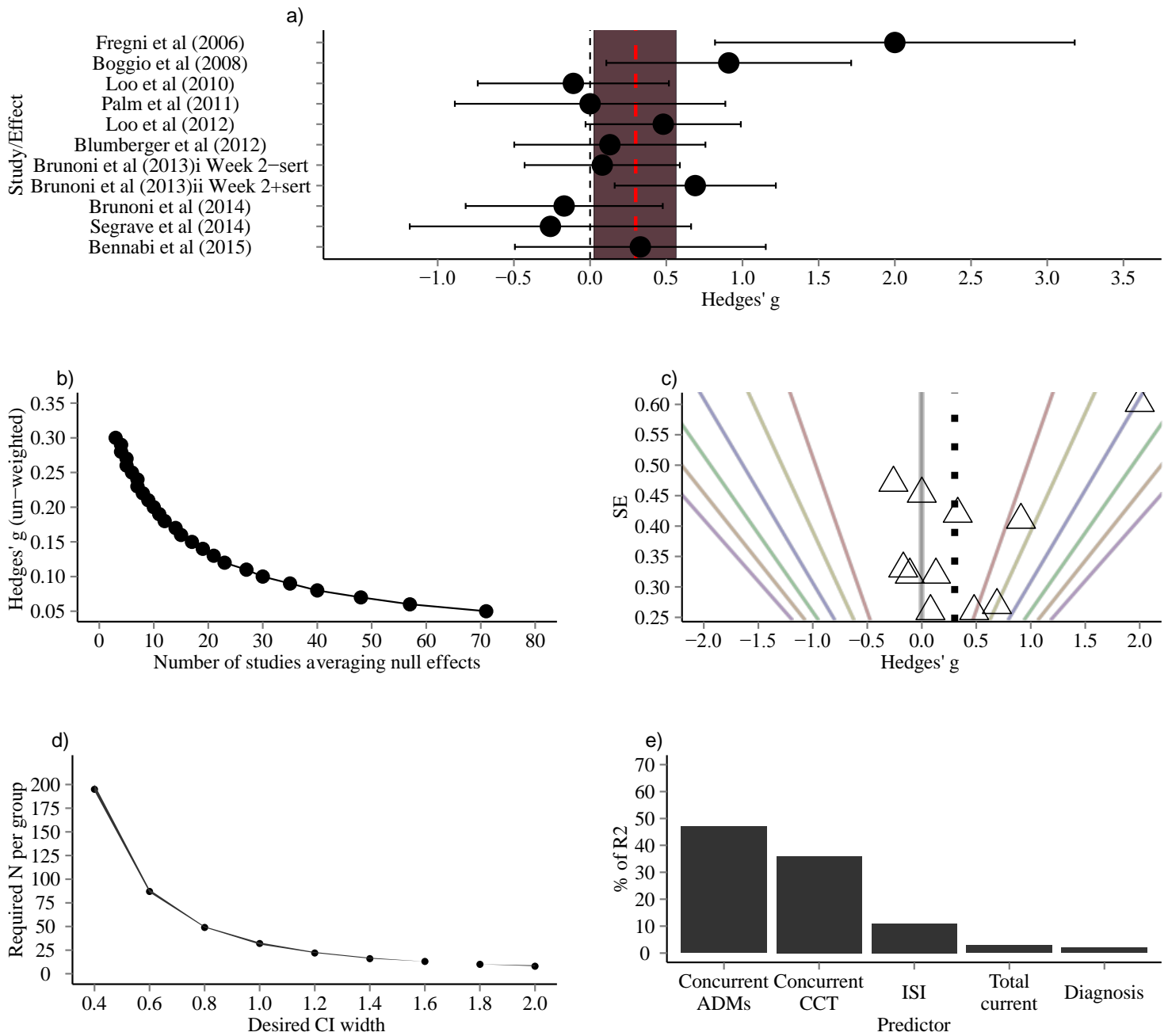

Figure 2. (a) Forest plot of effect sizes for active versus sham treatment. Error bars are $95 \%$ confidence intervals. Dotted red line depicts pooled effect. Shaded red region is $95 \%$ confidence interval. (b) Orwin fail-safe $\mathrm{N}$ analyses (c) Funnel plot. Dotted line is the pooled effect size, colored lines are $p$ values (e.g. red $=.05$, yellow $=.01$, blue $=.001$ ). (d) Outcome of precision analyses. For instance, to have $99 \%$ assurance that a $95 \% \mathrm{Cl}$ will be less than 0.8 , approximately 50 participants per group would be required. (e) Relative importance of each moderator. Note that total TDCS time, amplitude, session length and number of sessions were removed due to multi-colinearity. Total current is instead included to summarize these variables. 

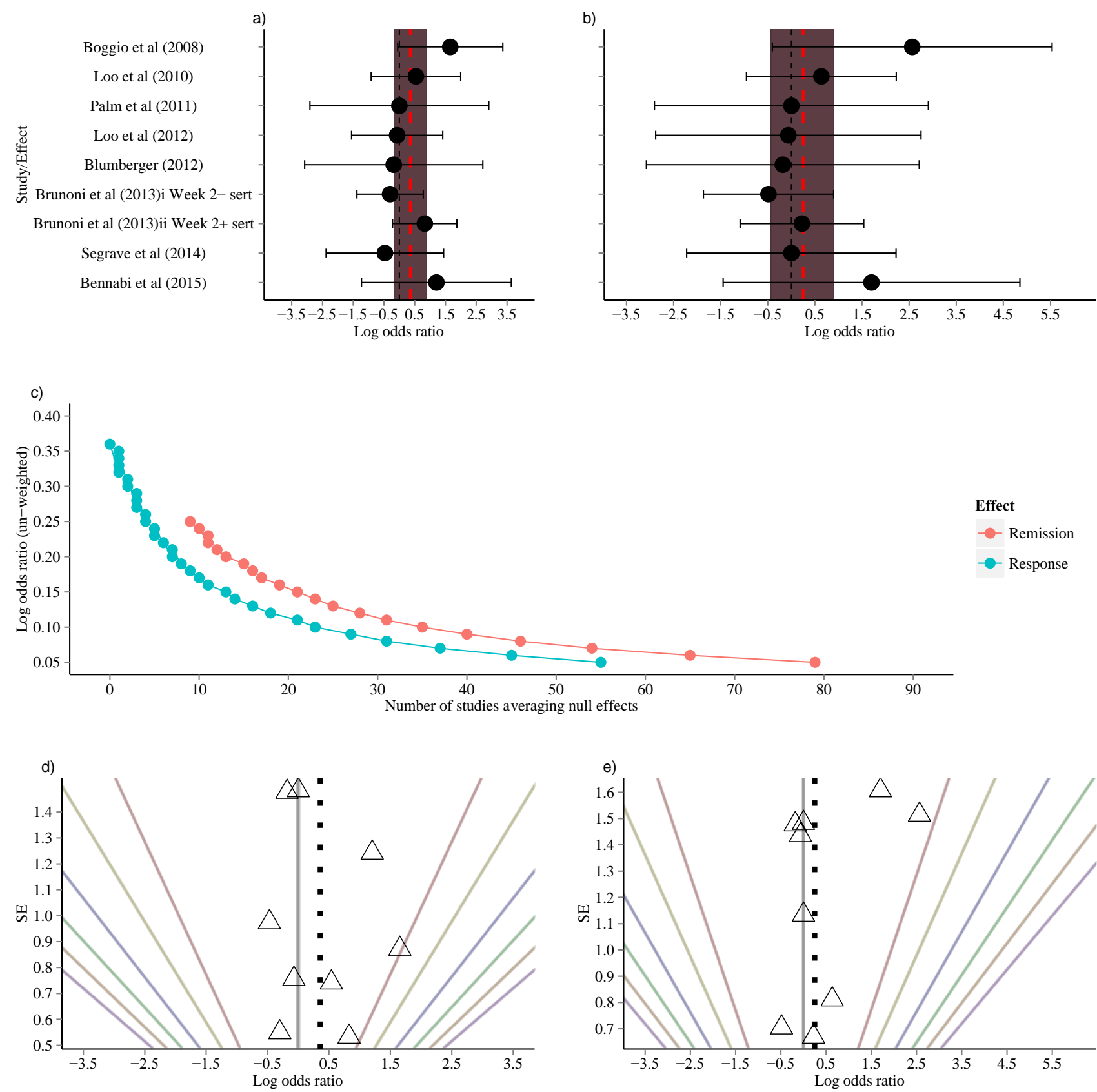

Figure 3. (a) Forest plot of effect sizes for active versus sham treatment: response rates. (b)

Forest plot of effect sizes for active versus sham treatment: remission rates. Error bars are

$95 \%$ confidence intervals. Dotted red line depicts pooled effect. Shaded red region is $95 \%$

confidence interval. (c) Orwin fail-safe $\mathrm{N}$ analyses (d) Funnel plot: response rates. (d) Funnel plot: remission rates. Dotted line is the pooled effect size, colored lines are p values (e.g. red $=.05$, yellow $=.01$, blue $=.001)$. 